\title{
Generating a Grid for Unstructured RANS Simulations of Jet Flows
}

Vance F. Dippold, III

Glenn Research Center, Cleveland, Ohio 


\section{NASA STI Program . . . in Profile}

Since its founding, NASA has been dedicated to the advancement of aeronautics and space science. The NASA Scientific and Technical Information (STI) Program plays a key part in helping NASA maintain this important role.

The NASA STI Program operates under the auspices of the Agency Chief Information Officer. It collects, organizes, provides for archiving, and disseminates NASA's STI. The NASA STI Program provides access to the NASA Technical Report Server-Registered (NTRS Reg) and NASA Technical Report ServerPublic (NTRS) thus providing one of the largest collections of aeronautical and space science STI in the world. Results are published in both non-NASA channels and by NASA in the NASA STI Report Series, which includes the following report types:

- TECHNICAL PUBLICATION. Reports of completed research or a major significant phase of research that present the results of NASA programs and include extensive data or theoretical analysis. Includes compilations of significant scientific and technical data and information deemed to be of continuing reference value. NASA counter-part of peer-reviewed formal professional papers, but has less stringent limitations on manuscript length and extent of graphic presentations.

- TECHNICAL MEMORANDUM. Scientific and technical findings that are preliminary or of specialized interest, e.g., "quick-release" reports, working papers, and bibliographies that contain minimal annotation. Does not contain extensive analysis.
- CONTRACTOR REPORT. Scientific and technical findings by NASA-sponsored contractors and grantees.

- CONFERENCE PUBLICATION. Collected papers from scientific and technical conferences, symposia, seminars, or other meetings sponsored or co-sponsored by NASA.

- SPECIAL PUBLICATION. Scientific, technical, or historical information from NASA programs, projects, and missions, often concerned with subjects having substantial public interest.

- TECHNICAL TRANSLATION. Englishlanguage translations of foreign scientific and technical material pertinent to NASA's mission.

For more information about the NASA STI program, see the following:

- Access the NASA STI program home page at http://www.sti.nasa.gov

- E-mail your question to help@sti.nasa.gov

- Fax your question to the NASA STI Information Desk at 757-864-6500

- Telephone the NASA STI Information Desk at 757-864-9658

- Write to:

NASA STI Program

Mail Stop 148

NASA Langley Research Center Hampton, VA 23681-2199 
NASA/TM-2018-219957

\section{Generating a Grid for Unstructured RANS Simulations of Jet Flows}

Vance F. Dippold, III

Glenn Research Center, Cleveland, Ohio

Prepared for the

Aviation Forum

sponsored by the American Institute for Aeronautics and Astronautics Atlanta, Georgia, June 25-29, 2018

National Aeronautics and

Space Administration

Glenn Research Center

Cleveland, Ohio 44135 


\section{Acknowledgments}

This work was supported by the NASA Transformational Tools and Technologies Project. The author is grateful for assistance from the FUN3D development team, in particular Jan-Renee Carlson.

This work was sponsored by the Transformative Aeronautics Concepts Program.

Trade names and trademarks are used in this report for identification only. Their usage does not constitute an official endorsement, either expressed or implied, by the National Aeronautics and Space Administration.

Level of Review: This material has been technically reviewed by technical management.

Available from

NASA STI Program

Mail Stop 148

NASA Langley Research Center

Hampton, VA 23681-2199
National Technical Information Service 5285 Port Royal Road Springfield, VA 22161

703-605-6000

This report is available in electronic form at http://www.sti.nasa.gov/ and http://ntrs.nasa.gov/ 


\title{
Generating a Grid for Unstructured RANS Simulations of Jet Flows
}

\author{
Vance F. Dippold, III \\ National Aeronautics and Space Administration \\ Glenn Research Center \\ Cleveland, Ohio 44135
}

\begin{abstract}
A study has been performed to determine best practices for generating unstructured grids for Reynolds-Averaged Navier-Stokes (RANS) simulations of jet flows. The Axisymmetric Near-Sonic Jet Case from the Turbulence Modeling Resource was used for this study: a Mach 0.985 flow through the 2-in. diameter Acoustic Reference Nozzle (ARN2). Simulations were run with FUN3D and used the Menter Shear Stress Transport (SST-V), Spalart-Allmaras (S-A), and $k$ - $k L$ turbulence models. The axial velocity and turbulent kinetic energy fields in the jet plume of the unstructured grid solutions were compared to those of the baseline structured grid solution provided by the Turbulence Modeling Resource. Only solutions using grids with structured-like elements in the jet plume showed good agreement with the baseline structured grid solution. Using the SST-V turbulence model, the fully unstructured grid solutions predicted the jet potential core to decay upstream of the baseline solution. With the S-A turbulence model, the unstructured grid solutions predicted the jet potential core to decay upstream of the baseline solution. The solutions using the $k-k L$ turbulence model seemed less sensitive to grid topology. Nozzle massflow and thrust performance were also compared for all simulations. Based on the results of this study, it is currently recommended that structured-like grid elements are used in the plumes of jet flows; unstructured grid elements can be used elsewhere.
\end{abstract}

\section{Nomenclature}

$\begin{array}{ll}A_{j e t} & \text { nozzle exit area } \\ a_{\infty} & \text { freestream speed of sound; } 1128 \mathrm{ft} / \mathrm{s} \\ C_{d} & \text { discharge coefficient } \\ C_{V} & \text { thrust coefficient } \\ D_{j e t} & \text { nozzle exit diameter; } 2.0 \mathrm{in} . \\ k & \text { turbulent kinetic energy } \\ M_{a} & \text { acoustic Mach number; } 0.90 \\ M_{\text {jet }} & \text { Mach number for ideally-expanded jet; } 0.985 \\ p & \text { pressure } \\ p_{\infty} & \text { freestream static pressure } \\ p_{0} & \text { nozzle total pressure } \\ r & \text { radial coordinate } \\ R_{j e t} & \text { radius of nozzle exit } \\ R_{\text {inflow }} & \text { radius at nozzle inflow } \\ T_{\infty} & \text { freestream static temperature } \\ T_{0} & \text { nozzle total temperature } \\ u & \text { streamwise velocity } \\ U_{j e t} & \text { ideally expanded jet velocity; } 1017 \mathrm{ft} / \mathrm{s} \\ x, y, z & \text { Cartesian coordinates } \\ y^{+} & \text {nondimensional wall distance }\end{array}$




$\begin{array}{ll}\rho & \text { density } \\ \rho_{\text {jet }} & \text { density of ideally expanded jet } \\ \theta & \text { azimuthal angle about } x \text {-axis }\end{array}$

\subsection{Introduction}

Structured grids have been widely used for Reynolds-Averaged Navier-Stokes (RANS) simulations of simple nozzle and jet flows. Whereas the jet plumes do not fully agree with experimental data in validation cases, the error is generally understood for a given type of jet flow and turbulence model. Furthermore, there are best practices for achieving a grid-converged solution. However, as nozzle geometries become increasingly complex (e.g., rectangular nozzles, embedded nozzles, plume-shock interactions), there is a greater demand to use unstructured grid generation methods. Unstructured grids offer greater flexibility than structured grids to create a computational grid around complex shapes. Relatively few authors have addressed the topic of generating unstructured grids for RANS simulations of jet flows. Pao et al. (Ref. 1) performed RANS simulations of two stream nozzles with structured and unstructured grids in order to predict jet noise. Unfortunately, there is rather limited discussion regarding the details of the unstructured grid generation and the results are mostly qualitative. However, Pao concluded that grid refinement is necessary in the shear layer, asserting that anisotropic grid cells (i.e., longer in the streamwise direction) may be most efficient in supporting gradients across shear layers.

A recent supersonic retropropulsion CFD validation effort explored the performance of unstructured grids (Refs. 2 to 4). Whereas this type of jet flow is a very difficult problem, it represents only a small subset of jet flow problems, so the applicability of the results to general jet flows may not be that strong. The solutions of several different unstructured flow solvers appeared to agree with each other and experimental data in qualitative comparisons of the flowfield. However, these reports did not present any quantitative comparisons of CFD solutions to each other and experimental data, nor did the reports present much regarding the details of the grids.

Most recently, Carter et al. (Ref. 5) performed unstructured grid simulations of two round convergentdivergent nozzle flows. The work used volume sources to generate the grid in the nozzle and plume, but the paper does not discuss grid cell sizes. The solutions agreed well with experimental data for both jets: centerline Mach and pressure profiles were presented for one jet flow; a nearfield pressure profile was presented for the second jet flow. A structured grid simulation was performed for one of the jets, but the unstructured and structured grid solutions did not agree well.

There is an apparent lack of documentation of the best practices for generating quality unstructured meshes for jet flows. The goal of this study is to determine a set of best practices for creating unstructured grids for RANS simulations of general jet flows that produce grid-independent solutions.

\subsection{Axisymmetric Near-Sonic Jet Flow}

The Axisymmetric Near-Sonic Jet Case from the Turbulence Modeling Resource (Ref. 6) was used for this study. The study uses the 2-in. diameter Acoustic Reference Nozzle (ARN2), which is a round, singlestream, convergent nozzle. The internal flowlines are pictured in Figure 1. For this study, the ARN2 was run using Set Point 7 from the Tanna Matrix, with a nozzle pressure ratio $\left(p_{0} / p_{\infty}\right)$ of 1.861 and nozzle temperature ratio $\left(T_{0} / T_{\infty}\right)$ of 1.0 into quiescent air. The freestream static pressure was set to 14.3 psi and the freestream static temperature was set to $530 \mathrm{R}$. These conditions correspond to an ideally expanded jet Mach number, $M_{j e t}$, of 0.985 and an ideally expanded jet velocity, $U_{j e t}$, of $1017 \mathrm{ft} / \mathrm{s}$. The acoustic Mach number, $M_{a}$, of the jet flow (equal to the ratio of the jet velocity, $U_{j e t}$, to the freestream speed of sound, $a_{\infty}$ ) was 0.90 . For the simulations, the freestream was only near-quiescent, with a Mach number set to 0.01. Ideally, the 


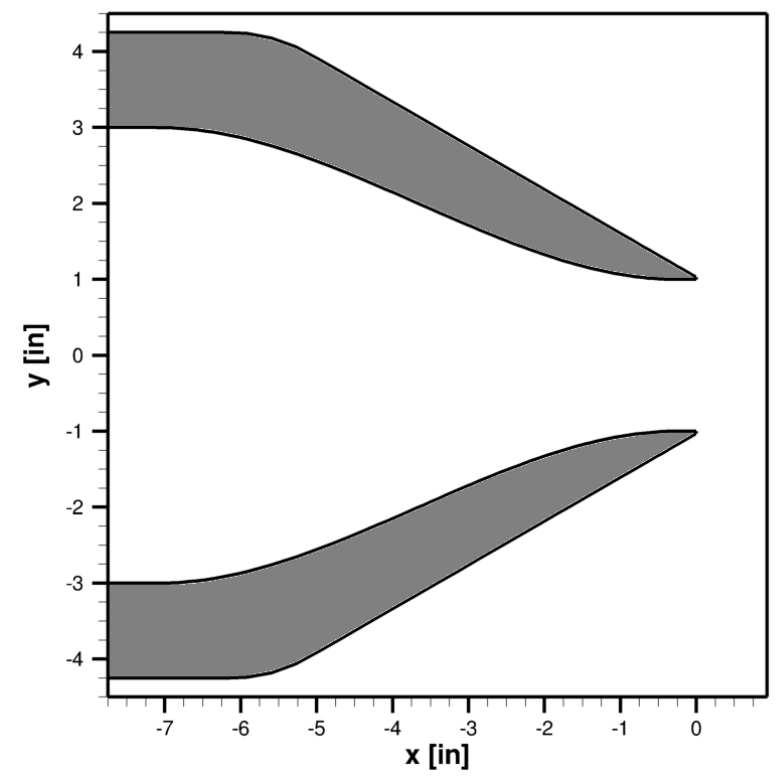

Figure 1.-Flowlines of the 2-in. diameter Acoustic Reference Nozzle.
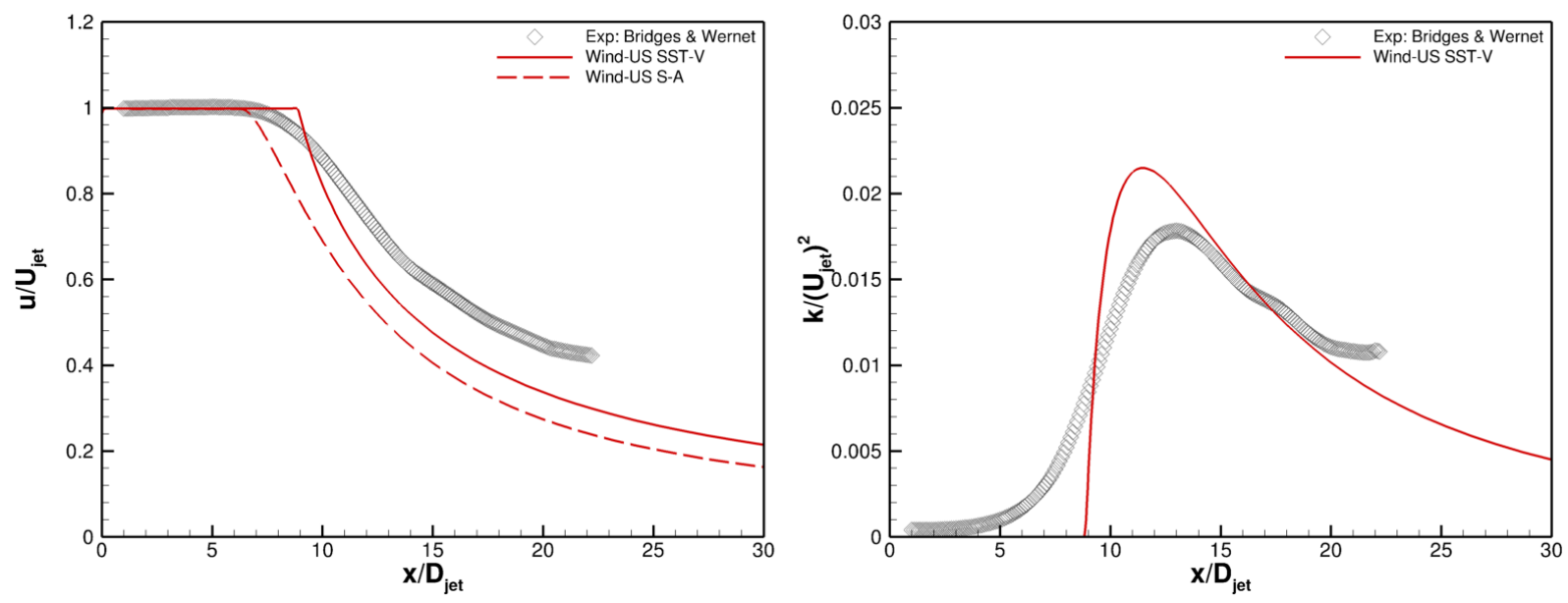

Figure 2.-Streamwise velocity, $u / \bigcup_{j e t}$, and turbulent kinetic energy, $k /\left(U_{j e t}\right)^{2}$, along the centerline of the ARN2 jet flow for experimental data and baseline structured solver solutions.

freestream would have a velocity of exactly zero, but compressible CFD codes can experience difficulties in convergence with a zero-flow freestream. Even a freestream Mach number as low as 0.01 helps the simulation converge more effectively and has minimal effect on the jet flow decay.

Experimental data for the ARN2 was reported by Bridges and Wernet (Refs. 7 and 8). The nondimensionalized streamwise velocity, $u / U_{j e t}$, and nondimensionalized turbulent kinetic energy, $k /\left(U_{j e t}\right)^{2}$, along the jet plume centerline are shown in Figure 2, denoted by the hollow diamond symbols. The jet potential core begins decaying (i.e., where $u<0.99 \times U_{\text {jet }}$ ) about $7.3 \times D_{\text {jet }}$ downstream of the nozzle exit. The peak value of turbulent kinetic energy along the centerline, $k /\left(U_{\text {jet }}\right)^{2}=0.0178$, occurs at $13.0 \times D_{\text {jet }}$ downstream of the nozzle exit. Streamwise velocity and turbulent kinetic energy at five stations through the plume are plotted in Figure 3. 

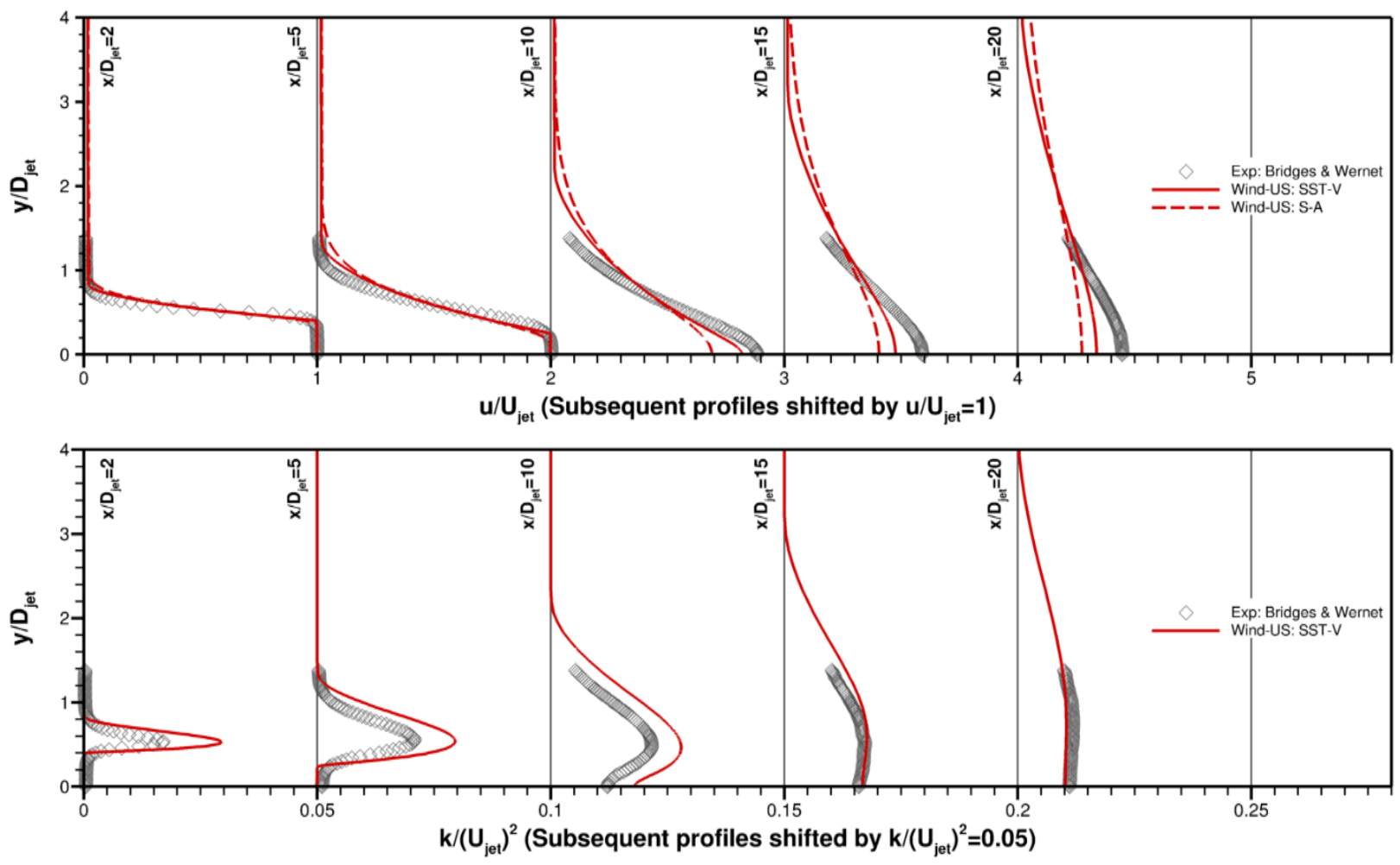

Figure 3.-Streamwise velocity, $u / \bigcup_{\text {jet }}$, (top) and turbulent kinetic energy, $k /\left(\bigcup_{j e t}\right)^{2}$, (bottom) at stations through the jet plume of the ARN2 experimental data and baseline structured solver cases.

The Turbulence Modeling Resource (Ref. 6) provides simulation data of the ARN2 jet flow from representative structured-grid RANS solvers. Structured grid solutions from the Wind-US flow solver (Ref. 9) are available using Menter's Shear Stress Transport turbulence model (Ref. 10) with vorticitybased production term (SST-V) and the Spalart-Allmaras turbulence model (Ref. 11) (S-A). Streamwise velocity (for the SST-V and S-A turbulence models) and turbulent kinetic energy (for the SST-V turbulence model only) along the jet centerline are plotted in Figure 2. The SST-V solution predicts a later decay of the jet core (by $1.66 \times D_{\text {jet }}$ ) than observed experimentally. The S-A solution predicts the jet core decay to be $0.63 \times D_{\text {jet }}$ earlier than observed experimentally. Both simulations (SST-V and S-A) predict a much steeper rate of jet core decay after the end of the potential core than observed experimentally. The SST-V solution predicts the peak turbulent kinetic energy along the jet centerline to occur $1.45 \times D_{j e t}$ earlier and be about 20 percent greater than what was observed experimentally. Profiles of streamwise velocity and turbulent kinetic energy at five stations in the jet plume from the structured-grid SST-V and S-A simulations are plotted in Figure 3. The profiles show the earlier jet core decay for both turbulence models at the $10 \times D_{\text {jet }}$ through $20 \times D_{\text {jet }}$ stations and the increased levels of turbulent kinetic energy for the SST-V turbulence model at the $2 \times D_{\text {jet }}$ through $10 \times D_{\text {jet }}$ stations. The Turbulence Model Resource shows that there is good agreement between the provided Wind-US solutions and solutions using the CFL3D flow solver for the SST-V and S-A turbulence models. Additionally, Jespersen, Pulliam, and Childs (Ref. 12 show good agreement between OVERFLOW and the provided Wind-US solutions for both turbulence models. The solutions from both turbulence models (SST-V and S-A) show clear discrepancies with the experimental data. However, as the Turbulence Modeling Resource states, 
the goal is to provide a set of test cases that illustrate the performance of models for flows that capture fundamental phenomena, in order to establish a consistent basis of comparison as a starting point from which a more thorough validation effort for flows of specific interest to users and developers can be conducted. (Ref. 6)

Therefore, it can be assumed that grid-converged solutions should be similar for a given turbulence model. The structured grid SST-V and S-A turbulence model solutions for the Axisymmetric Near-Sonic Jet Case will be used as a baseline to assess the unstructured grid RANS solutions explored in this study.

\subsection{Numerical Simulations}

\subsection{FUN3D Flow Solver}

FUN3D (Ref. 13), a production flow solver and design code developed at the NASA Langley Research Center, was selected as the flow solver for the simulations in this study. FUN3D is a nodebased, finite volume Navier-Stokes solver and can solve both compressible and incompressible flow problems. FUN3D version 12.9 was used for this study. Each simulation used the Roe flux difference splitting scheme (Ref. 14) for the inviscid flux residual construction and the Van Leer flux-vector splitting scheme (Ref. 15) for the left hand side inviscid flux Jacobian construction. Additionally, all grids tested in this study were run using the two-equation Menter Shear Stress Transport turbulence model (Ref. 10) with vorticity-based source term (SST-V). Selected grids were also run using the single-equation SpalartAllmaras turbulence model (Ref. 11) (S-A) and the $k-k L$ turbulence model (Ref. 16).

\subsection{Boundary Conditions}

The boundary conditions and initial conditions are input into FUN3D based on a reference set of conditions. For all cases, the freestream conditions were used as the reference conditions. The jet flow is exhausted into a near-quiescent freestream, with a Mach number of 0.01 . The near-sonic jet simulations presented on the Turbulence Modeling Resource (Ref. 6) also used a freestream Mach number of 0.01. The freestream static temperature was set to $530 \mathrm{R}$. Using a freestream static pressure of $14.3 \mathrm{psi}$, the freestream Reynolds number was set to 5789/in. At the nozzle inflow, the nozzle temperature ratio, $T_{0} / T_{\infty}$, was set to 1.000 , corresponding to a jet total temperature of $530 \mathrm{R}$; the nozzle pressure ratio, $p_{0} / p_{\infty}$, was set to 1.861, corresponding to a jet total pressure of 26.612 psi. FUN3D's subsonic inflow boundary condition (7011 in FUN3D) was applied to the nozzle inflow. The freestream boundary condition (5050 in FUN3D) was applied to the inflow boundary external to the nozzle. The farfield boundary condition (5000 in FUN3D) was applied to the farfield boundaries, the boundaries radially farthest from the jet centerline. FUN3D's back pressure boundary condition (5051in FUN3D) was applied to the outflow of the computational domain. The viscous wall boundary condition (4000in FUN3D) was applied to the nozzle's interior and exterior viscous surfaces. The viscous walls were treated as adiabatic. A $180^{\circ}$ sector of the jet flow was modeled, with the symmetry plane along the $z=0$ plane. The appropriate symmetry boundary condition was used (6663in FUN3D).

\subsection{Simulation Convergence}

All simulations were completed on the multinode, multicore NASA Advanced Supercomputing (NAS) Pleiades supercomputer (Ref. 17). Each simulation used between 20 and 400 processor cores (depending upon the grid size). The simulations were run as steady-state, with a global CourantFriedrichs-Lewy (CFL) number. For nearly all cases, the CFL number was increased from a value of 
0.5 at start-up to a final CFL number of 5.0 or 10.0. Several of the cases did not monotonically converge using a global CFL number. For these cases, the simulations were run time-accurately, with a constant time step. FUN3D uses a dual time-stepping method: the time-accurate simulations used a dimensional time step of $1 \times 10^{-5}$ seconds and five subiterations per time step with a CFL number of 5.0.

As the simulations were performed, convergence was monitored in multiple ways. The residuals of the RANS and turbulence model equations were monitored. For converged solutions, the residuals had to decrease by two to four orders of magnitude. Second, the solution flowfield was monitored, specifically the streamwise velocity and the turbulent kinetic energy through the jet plume. Once the flowfield showed no significant change, the solution was deemed converged.

\subsection{Grids}

The objective of this study was to determine best practices for generating unstructured grids for jet flows. Twenty-four different grids were generated for this study, in an attempt to determine techniques and topologies that produced solutions that most closely agreed with the baseline structured grid solutions from the Turbulence Modeling Resource. All the grids in this study were generated using Pointwise ${ }^{\circledR}$ (Ref. 18) and modeled only a $180^{\circ}$ sector of the jet flow.

\subsection{Structured-Like Grids}

The first grids generated were structured-style grids: grids that used hexahedral elements arranged in a topology like a typical structured grid. (For the purposes of this work, hexahedral grid elements refer to grid elements that are generally rectangular prisms.) The purpose of running simulations with structuredlike grids was to validate the FUN3D solver against the baseline structured grid solutions. The Turbulence Modeling Resource (Ref. 6) provides four three-block, structured, 2D grids for axisymmetric simulations of the ARN2 nozzle. These grids range in size from 4,730 nodes to 280,517 nodes. Case 1a rotationally extruded the second finest grid (with block sizes of $97 \times 97$ nodes inside the nozzle and $257 \times 225$ nodes downstream of the nozzle) 112 steps about the jet centerline to form a $180^{\circ}$ sector.

The symmetry plane of the Case 1a grid is pictured in Figure 4. The Case 1a grid had approximately 8.2 million nodes. The Case 1a grid had an O-grid topology, as pictured in Figure 5(a).

Because the cells along the jet centerline have very small volumes and can cause some CFD codes to converge slowly, the Case $1 \mathrm{~b}$ grid replaced the O-grid near the centerline with an H-grid to increase the size of the grid cells near the centerline. The Case $1 \mathrm{~b}$ grid at the nozzle exit plane is pictured in Figure 5(b). The Case 1b grid had approximately 8.1 million nodes.

The Case 1c grid, a coarser version of the Case 1a grid, extruded the third finest grid from the Turbulence Modeling Resource (with block sizes of 49×49 nodes inside the nozzle and 129×113 nodes downstream of the nozzle) 56 steps about the jet centerline to form a $180^{\circ}$ sector. The Case $1 \mathrm{c}$ grid contained about 1.07 million nodes.

\subsection{General Unstructured Grid Properties}

As mentioned, all the unstructured grids for this study were generated using Pointwise ${ }^{\circledR}$ version 17.3R4 (Ref. 18). Symmetry was used and only a $180^{\circ}$ sector of the flow was modeled. The normal wall spacing was $0.0002 \mathrm{in}$. along the interior of the nozzle and $0.002 \mathrm{in}$. along the exterior of the nozzle. These normal wall spacings were similar to those used for the second finest grid on the Turbulence Modeling Resource (and in the Case 1a and Case 1b grids). Based on the skin friction near the nozzle exit, the $y^{+}$value at the wall ranged between four and five for most grids. In many of the grids, 


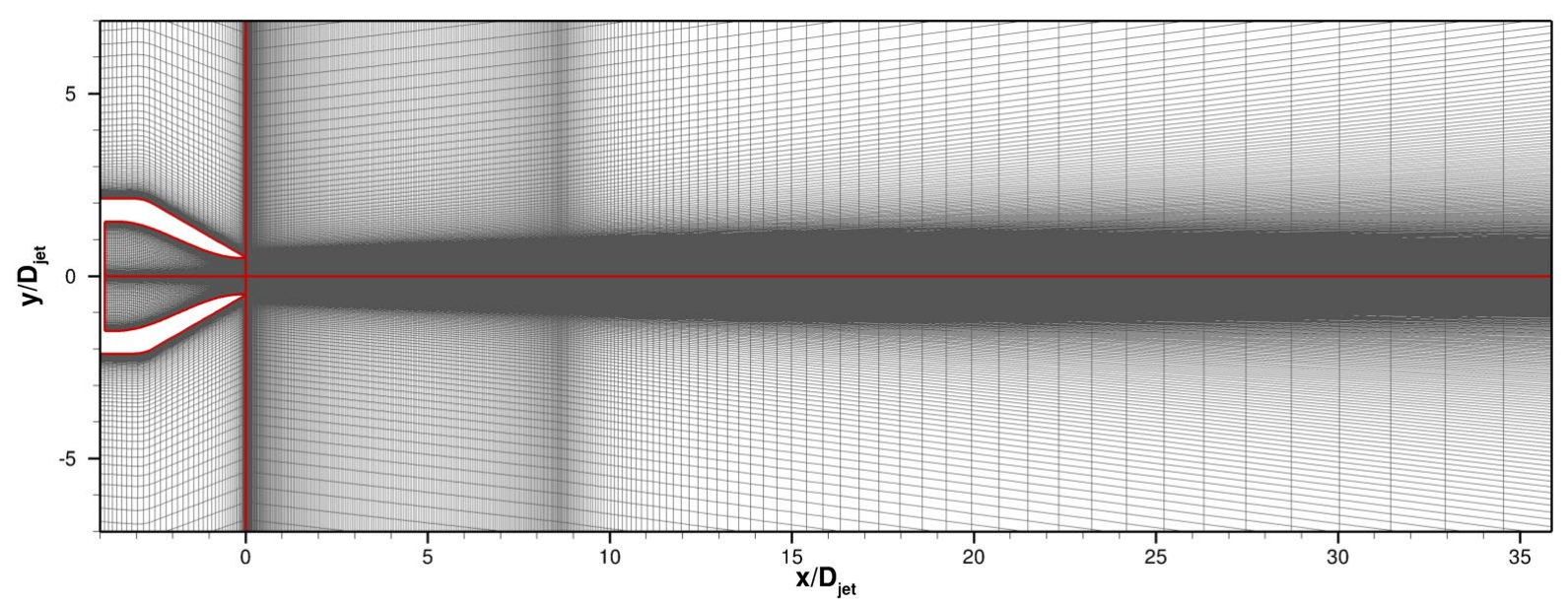

Figure 4.-The Case 1a grid, pictured along the symmetry plane. Red lines denote block edges.

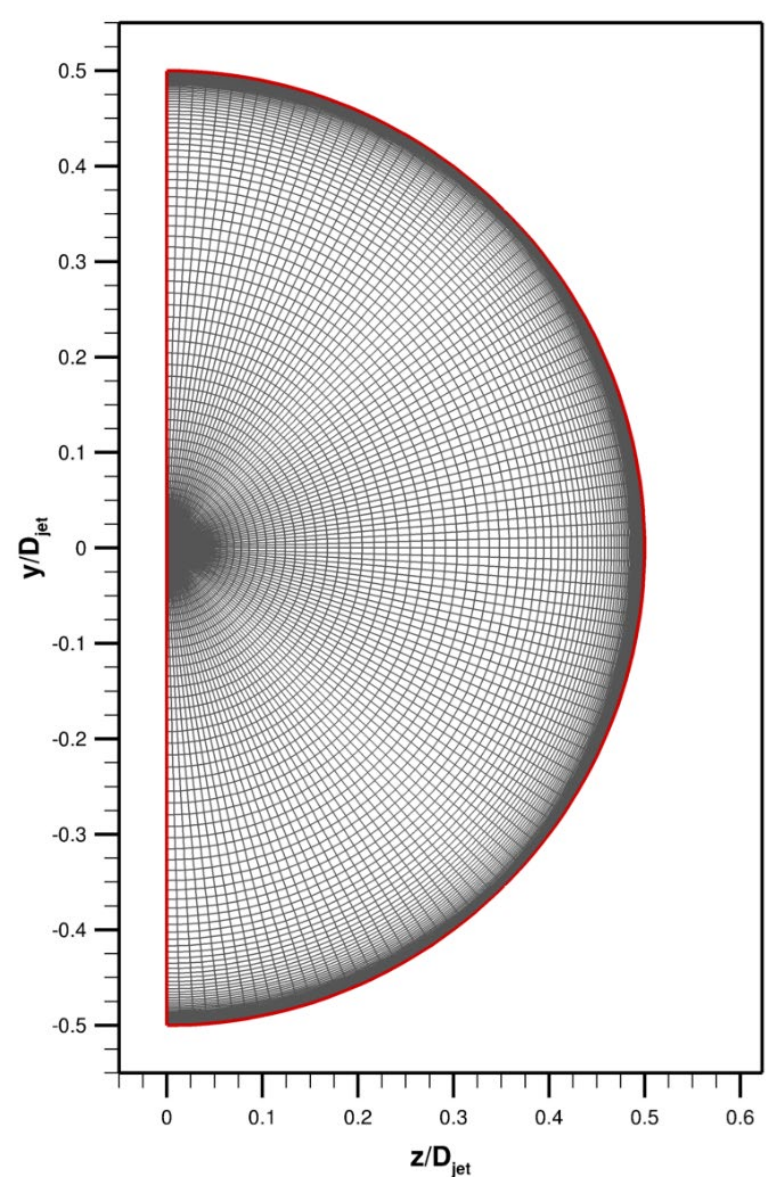

(a) Case 1a: O-grid topology along centerline.

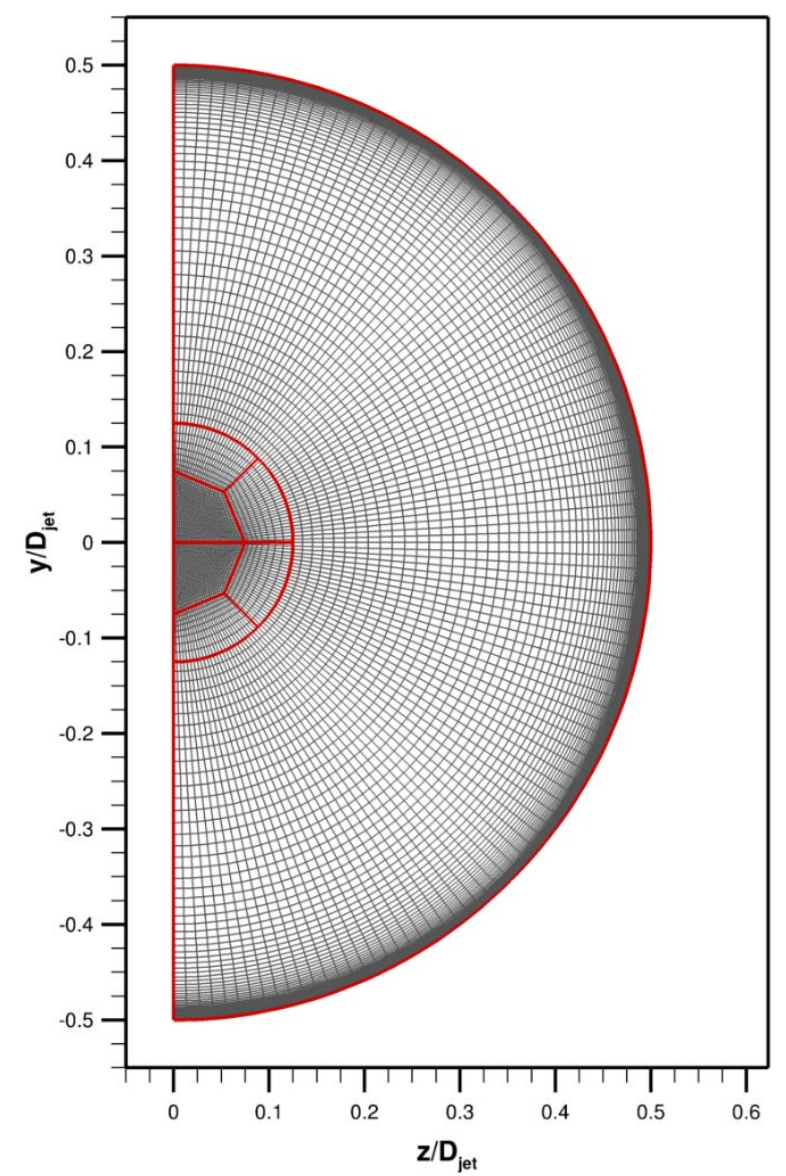

(b) Case $1 \mathrm{~b}$ : H-grid topology along centerline. Figure 5.-Grid topology at nozzle exit plane of the Case $1 \mathrm{a}$ and Case $1 \mathrm{~b}$ grids. 
a block interface was placed in the jet plume, parallel to the jet centerline, to serve as a baffle (i.e., source of refinement) surface. The radial spacing along the baffle surface was $0.02 \mathrm{in}$. for most of the unstructured grids. Surface spacing along the nozzle surfaces and the baffle surfaces was generally 0.03 in. On the nozzle and baffle surfaces, the unstructured boundary decay factor (which determines the influence that the boundary spacing has on the interior spacing of the surface or volume grid) was set to 0.99. In the volume grid, the unstructured boundary decay factor was set between 0.9 and 0.99 . The anisotropic tetrahedral extrusion (T-Rex) advancing layer technique within Pointwise ${ }^{\circledR}$ was used to generate the grid in a structured-like manner near the viscous and baffle surfaces. Typically, the grids used a T-Rex growth rate factor of 1.3 and a T-Rex maximum angle of $165^{\circ}$.

\subsection{First Series of Unstructured Grids}

In the first unstructured grid created for the ARN2 jet flow-the Case 20 grid—grid points were clustered about the centerline using the same streamwise spacing used in the baseline structured grids. The Case 20 grid was composed entirely of tetrahedral elements and had approximately 0.5 million nodes. It is pictured in Figure 6. The next four grids generated-Case 2a through Case 2d-were also composed entirely of tetrahedral elements. Grid points were clustered along a baffle surface with a diameter of $1 \times D_{j e t}$, extending downstream of the nozzle exit for lengths of $0 \times D_{j e t}, 5 \times D_{j e t}, 10 \times D_{j e t}$, and $20 \times D_{j e t}$. These grids ranged in size from about 0.46 million nodes to 3.0 million nodes. Details of the Case 2a through Case 2d grids are listed in Table I. Figure 7 shows the grid near the nozzle exit for the Case $2 \mathrm{a}$ and the Case $2 \mathrm{~d}$ grids.

The Combine Anisotropic Tetrahedra feature within Pointwise ${ }^{\circledR}$ was used to create a new set of grids based on the Case 20 and Case 2a through Case 2d grids. This feature combines the anisotropic tetrahedral cells created by the T-Rex solver into hexahedral elements to more closely resemble structured grid cells. A secondary effect of combining the anisotropic tetrahedral cells is that the number of grid cells is reduced whereas the number of grid nodes remains the same. The new grids were named the Case 30 and Case 3a through Case 3d grids. Further details are given in Table I.

\subsection{Second Series of Unstructured Grids}

Simulations of the Case 20 and Case 2a through Case 2d grids indicated that further grid refinement was necessary. In the Case 2e through Case $2 \mathrm{i}$ grids, cells were clustered along the centerline and constant diameter baffle surface $\left(1 \times D_{j e t}\right)$, with a constant grid spacing of 0.03 in. The length of the refinement within the jet plume varied: $10 \times D_{\text {jet }}, 15 \times D_{\text {jet }}, 20 \times D_{\text {jet }}, 25 \times D_{\text {jet }}$, and $30 \times D_{\text {jet }}$ downstream of the nozzle exit plane. These grids ranged in size from 2.1 to 5.2 million nodes. The grid near the nozzle exit of the Case $2 \mathrm{i}$ grid is pictured in Figure 8 . The Case $2 \mathrm{j}$ grid was very similar to the Case $2 \mathrm{i}$ grid. The only difference was that the Case $2 \mathrm{j}$ grid used the Advancing Front surface grid algorithm rather than the Delaunay surface grid algorithm, which had been used in all previously created tetrahedral grids. As can be observed in Figure 9, the Advancing Front algorithm generates a surface grid that looks more uniform than the surface grid generated by the Delaunay algorithm. All subsequent unstructured grids used the Advancing Front surface grid algorithm. The Case 2j grid had 5.3 million nodes. 


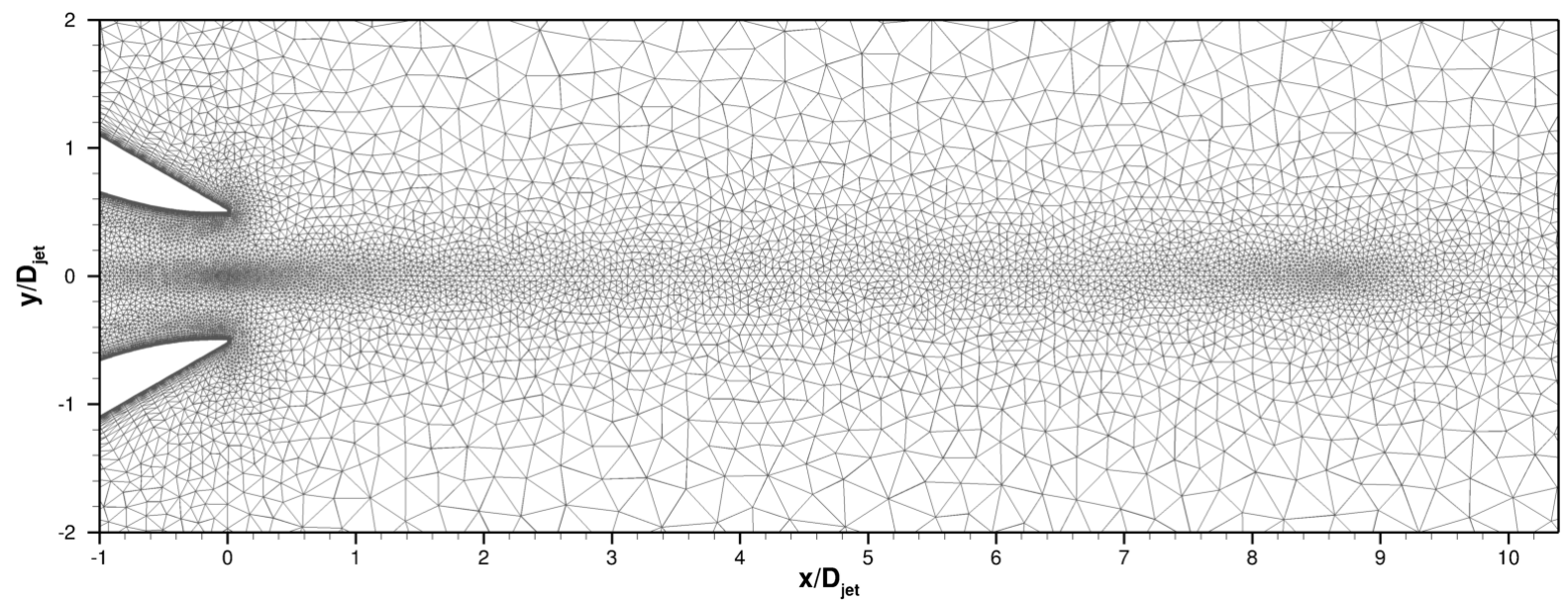

Figure 6.-The Case 20 grid near the nozzle exit, pictured along the symmetry plane.

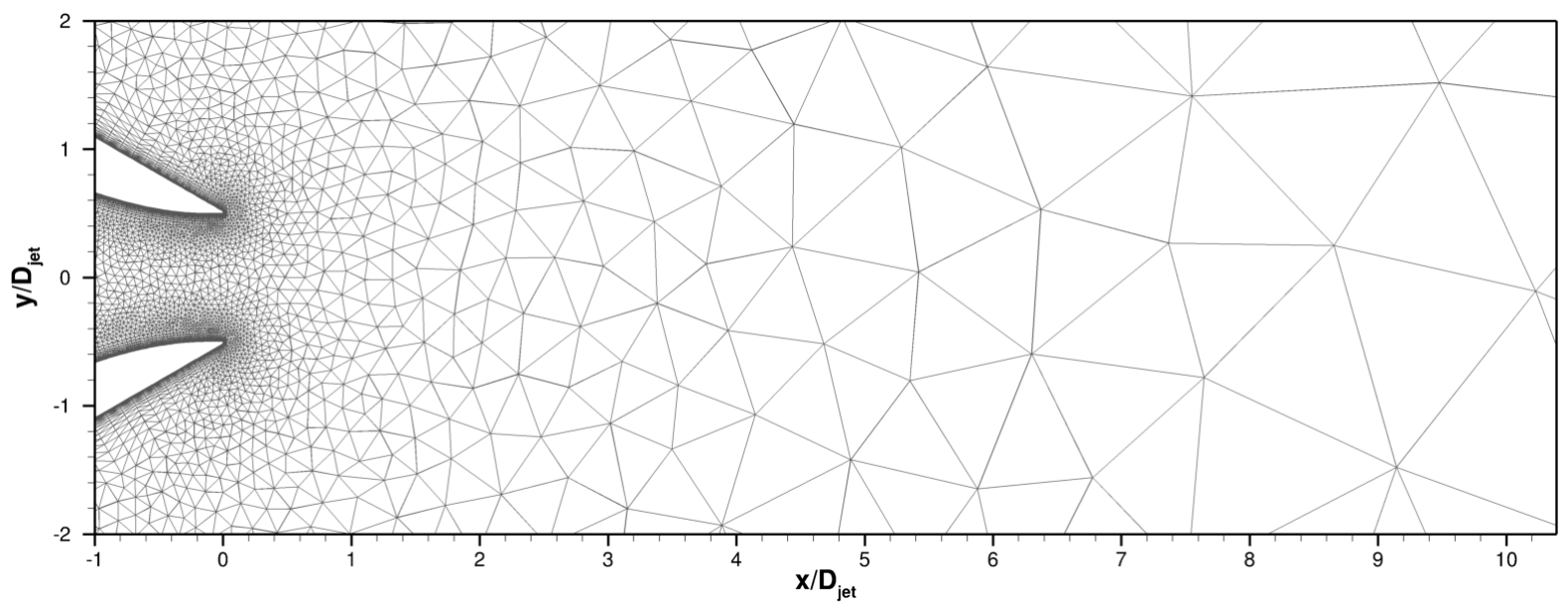

(a) Case 2a.

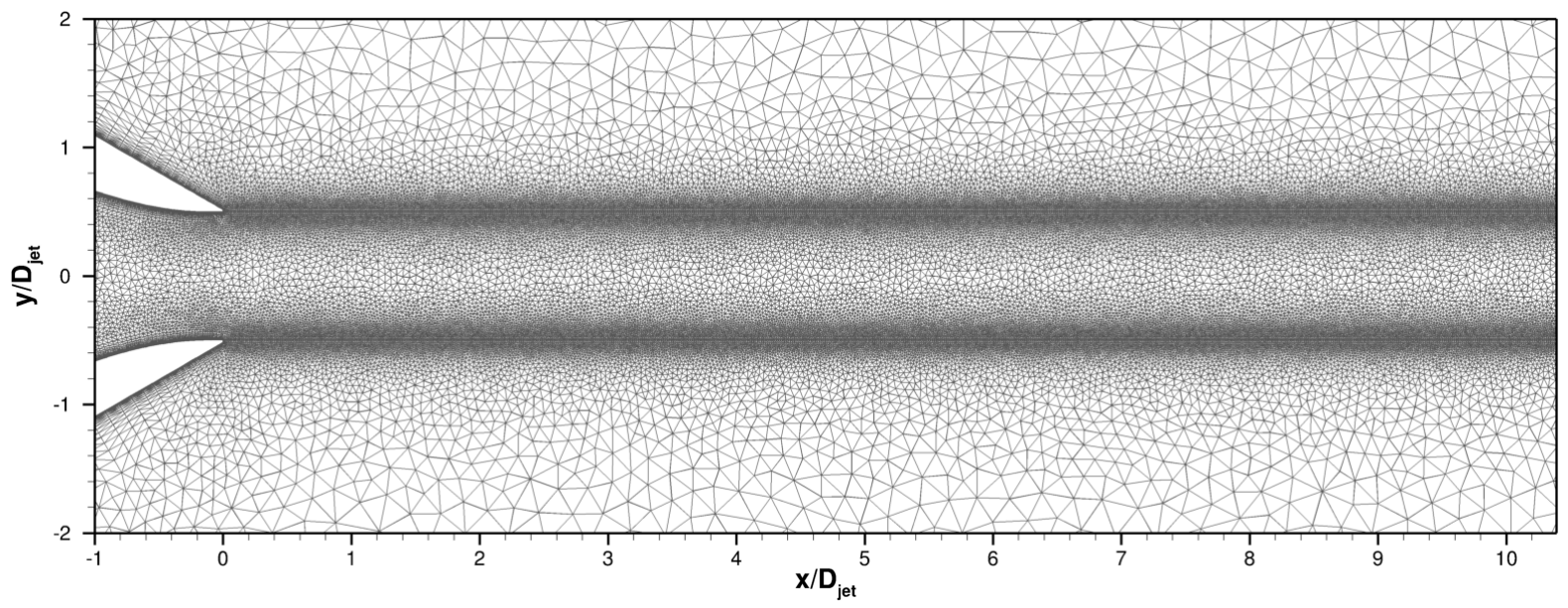

(b) Case $2 \mathrm{~d}$.

Figure 7.-The Case $2 \mathrm{a}$ and Case $2 \mathrm{~d}$ grids near the nozzle exit, pictured along the symmetry plane. 
TABLE I.-GRID TOPOLOGY DETAILS

\begin{tabular}{|c|c|c|c|c|}
\hline Grid & Total nodes & Plume grid length & Grid cell elements & Notes \\
\hline Case 1a & $8,204,605$ & N/A & Hexahedra & O-grid along CL \\
\hline Case $1 \mathrm{~b}$ & $8,149,537$ & N/A & Hexahedra & H-grid along CL \\
\hline Case $1 c$ & $1,066,415$ & N/A & Hexahedra & O-grid along CL \\
\hline Case 20 & 498,026 & Centerline & Tetrahedra & \\
\hline Case $2 \mathrm{a}$ & 457,643 & $0 \times D_{j e t}$ & Tetrahedra & No CL refinement \\
\hline Case 2b & $1,158,638$ & $5 \times D_{j e t}$ & Tetrahedra & No CL refinement \\
\hline Case $2 c$ & $1,806,012$ & $10 \times D_{j e t}$ & Tetrahedra & No CL refinement \\
\hline Case 2d & $3,053,378$ & $20 \times D_{\text {jet }}$ & Tetrahedra & No CL refinement \\
\hline Case $2 \mathrm{e}$ & $2,123,345$ & $10 \times D_{j e t}$ & Tetrahedra & \\
\hline Case $2 \mathrm{f}$ & $2,863,400$ & $15 \times D_{\text {jet }}$ & Tetrahedra & \\
\hline Case $2 \mathrm{~g}$ & $3,691,425$ & $20 \times D_{\text {jet }}$ & Tetrahedra & \\
\hline Case $2 \mathrm{~h}$ & $4,401,710$ & $25 \times D_{\text {jet }}$ & Tetrahedra & \\
\hline Case $2 \mathrm{i}$ & $5,213,065$ & $30 \times D_{\text {jet }}$ & Tetrahedra & \\
\hline Case $2 \mathrm{j}$ & $5,292,462$ & $30 \times D_{\text {jet }}$ & Tetrahedra & Advancing front \\
\hline Case $2 \mathrm{k}$ & $53,375,821$ & $30 \times D_{\text {jet }}$ & Tetrahedra & Advancing front \\
\hline Case 30 & 498,026 & Centerline & Mixed & Case 20 with CAT \\
\hline Case 3a & 457,643 & $0 \times D_{\text {jet }}$ & Mixed & Case 2a with CAT; No CL refinement \\
\hline Case 3b & $1,158,638$ & $5 \times D_{\text {jet }}$ & Mixed & Case 2b with CAT; No CL refinement \\
\hline Case 3c & $1,806,012$ & $10 \times D_{j e t}$ & Mixed & Case 2c with CAT; No CL refinement \\
\hline Case 3d & $3,053,378$ & $20 \times D_{j e t}$ & Mixed & Case 2d with CAT; No CL refinement \\
\hline Case 40 & $26,619,632$ & $30 \times D_{\text {jet }}$ & Hybrid & H-grid along CL \\
\hline Case 50 & $28,273,154$ & $30 \times D_{j e t}$ & Tetrahedra & Advancing Front \\
\hline Case 6a & $8,315,666$ & $30 \times D_{\text {jet }}$ & Triangular prisms & O-grid along CL \\
\hline Case 6b & $8,315,666$ & $30 \times D_{j e t}$ & Triangular prisms & O-grid along CL \\
\hline
\end{tabular}

Legend $\quad$ CAT $=$ Combined Anisotropic Tetrahedra

$\mathrm{CL}=$ Centerline

Mixed (elements) = Hexahedra, tetrahedra, and pyramids

Advancing Front = surface grid algorithm (Delaunay surface grid algorithm used for Case 20, Case 2a-

Case 2i, Case 30, Case 3a-Case 3d)

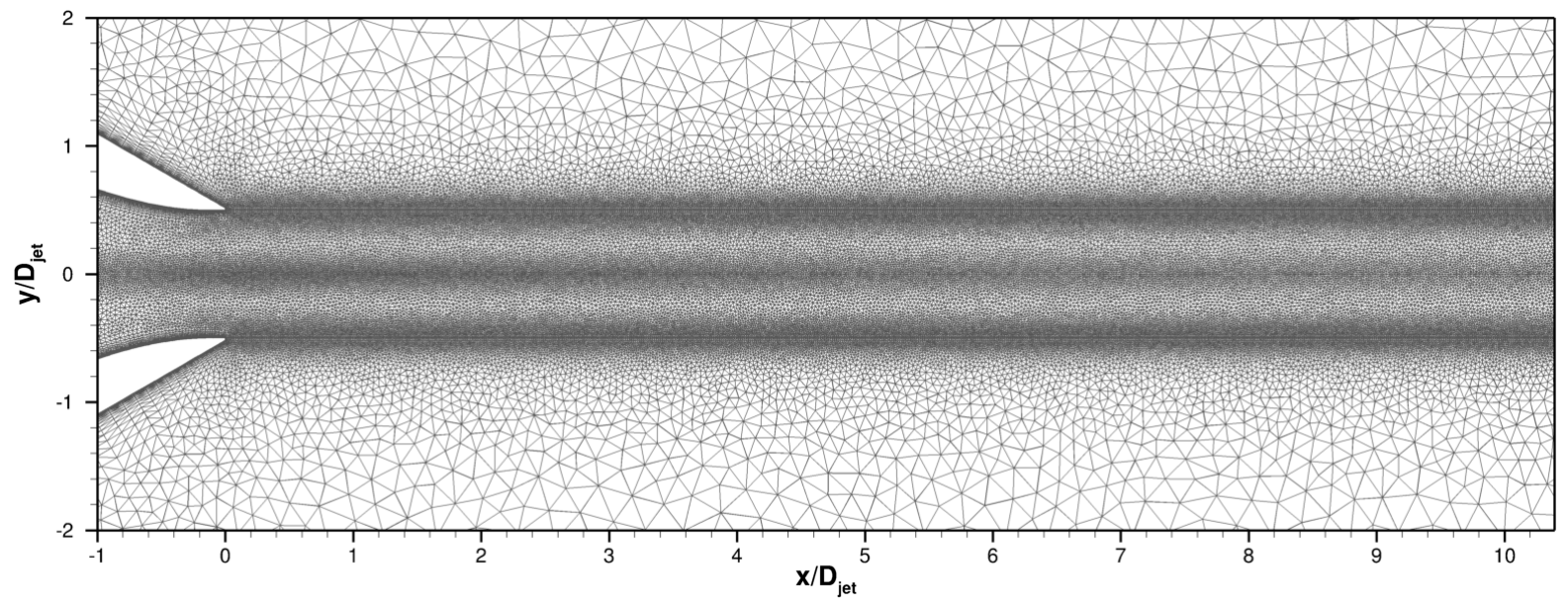

Figure 8.-The Case $2 \mathrm{i}$ grid near the nozzle exit, pictured along the symmetry plane. 


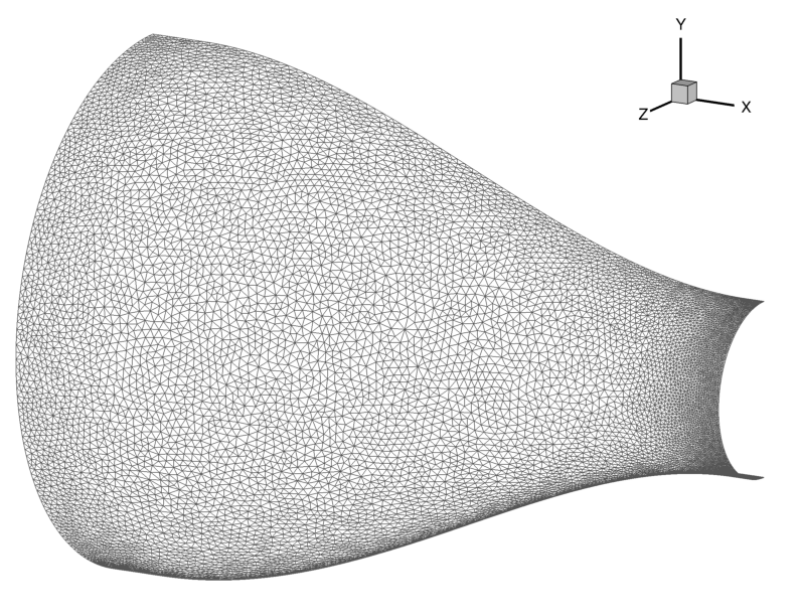

(a) Case 2i: Delaunay algorithm.

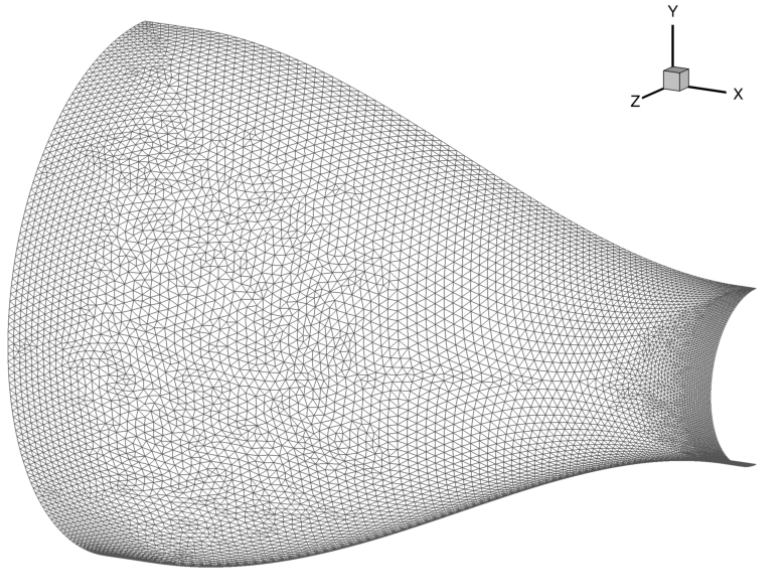

(b) Case 2j: Advancing Front algorithm.

Figure 9.-Comparing the Delaunay and Advancing Front surface grid algorithms. Grid shown for the nozzle interior surface.

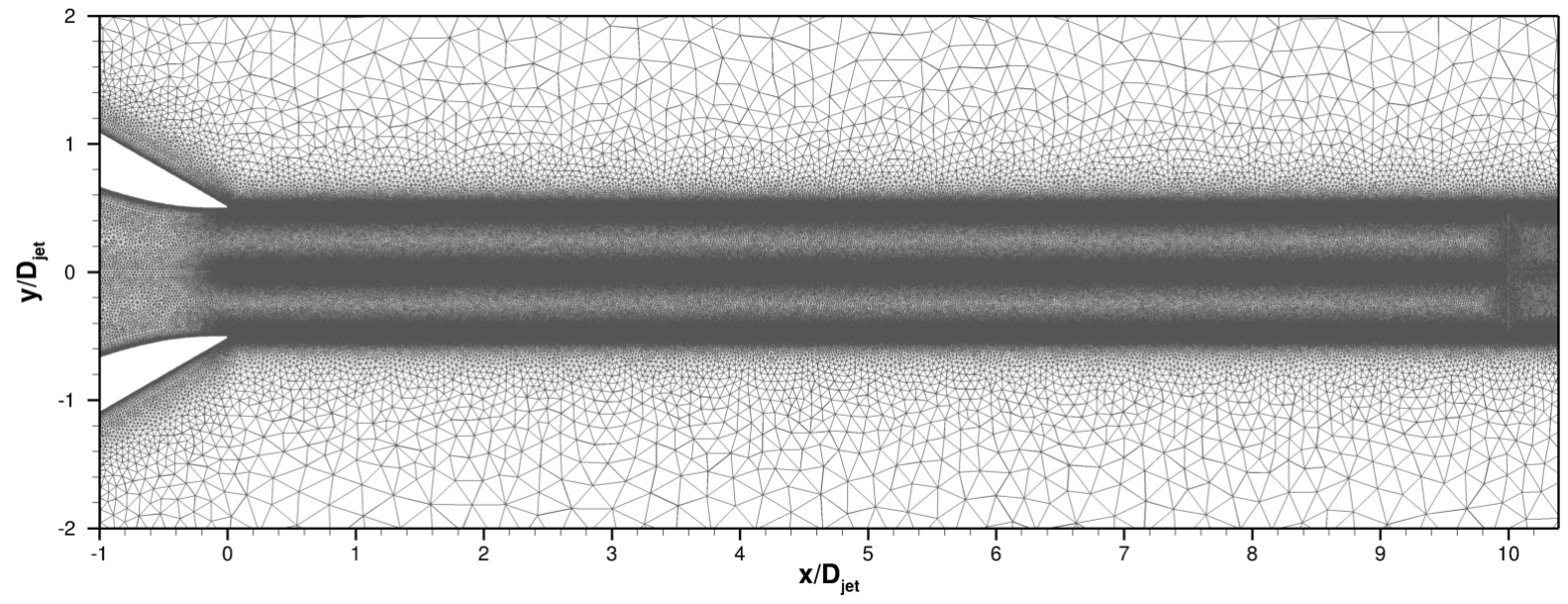

Figure 10.-The Case $2 \mathrm{k}$ grid near the nozzle exit, pictured along the symmetry plane.

\subsection{Extra Fine Unstructured Grid}

A finer all-tetrahedral element grid was generated, the Case $2 \mathrm{k}$ grid. The surface spacing along the nozzle interior surface of the previously created tetrahedral grids varied from $0.1 \mathrm{in}$. at the nozzle inflow to $0.03 \mathrm{in}$. at the nozzle exit. The nozzle interior surface grid of the Case $2 \mathrm{k}$ grid was a constant $0.05 \mathrm{in}$. from the nozzle inflow to $0.5 \times D_{\text {jet }}$ upstream of the exit, at which point it transitioned to $0.01 \mathrm{in}$. at the nozzle exit. Surface spacing along the plume baffle surface and along the jet centerline was also set to $0.01 \mathrm{in}$., rather than 0.03 in. as used in earlier tetrahedral grids. The radial grid spacing along the jet plume baffle was reduced from 0.02 to $0.01 \mathrm{in}$. The jet plume refinement region extended $30 \times D_{\text {jet }}$ downstream of the nozzle exit. The Case 2k grid had 53.4 million nodes. The grid is pictured in Figure 10. 


\subsection{Hybrid Grid}

A hybrid grid was generated, blending a structured-like grid in the jet flow with an unstructured grid in the surrounding flowfield. The Case 40 grid was composed of a structured-like grid in the nozzle and jet plume similar to the Case 1b grid: an H-grid along the centerline, surrounded by an O-grid. The structured-like grid extended $30 \times D_{\text {jet }}$ downstream of the nozzle exit, with constant diameter of $1 \times D_{j e t}$. The streamwise grid spacing was set to 0.03 in. near the nozzle exit and through the entire length to the jet plume region; this was similar to the streamwise grid spacing used at the nozzle exit in the Case 1a and Case $1 \mathrm{~b}$ grids. Because the structured-like grid would be blended with an unstructured tetrahedral element grid, care was taken to minimize streamwise grid cell stretching along the shear layer so that the grids could be blended together smoothly. The radial grid spacing of the Case 40 grid also used the same radial grid spacing as the Case 1a and Case 1b grids in the nozzle, with a normal wall spacing of 0.0002 in. along the nozzle interior. Like the Case $1 \mathrm{a}$ and Case $1 \mathrm{~b}$ grids, the normal wall spacing along the nozzle exterior was set to 0.002 in. The radial spacing at the interface between the structured-like plume grid and the unstructured outer flowfield grid was 0.002 in. The T-Rex grid solver was used within Pointwise ${ }^{\circledR}$ to blend the structured-like jet plume region with the unstructured outer flowfield. Pyramid elements were used in the region near the structured-like plume grid to help smoothly blend the grid from hexahedral elements to tetrahedral elements. The grid consisted of 26.6 million nodes and is pictured in Figure 11. The Case 40 grid does have significantly more grid points than either the structured Case $1 \mathrm{~b}$ grid (8.1 million grid nodes), and the unstructured Case $2 \mathrm{j}$ grid (5.3 million grid nodes). In the jet plume, the structured grids used grid-stretching in the streamwise direction, whereas the fully tetrahedral unstructured grids used a constant grid spacing. The structured-like jet plume of the hybrid grid used a constant grid spacing in the streamwise direction in order to smoothly blend into the surrounding unstructured flowfield. But, like a structured grid, the plume region was much finer in the radial direction than the Case $2 \mathrm{j}$ grid. This caused the jet plume region of the Case 40 grid to have three to five times as many grid nodes as found in the Case $1 \mathrm{~b}$ and the Case $2 \mathrm{j}$ grids.

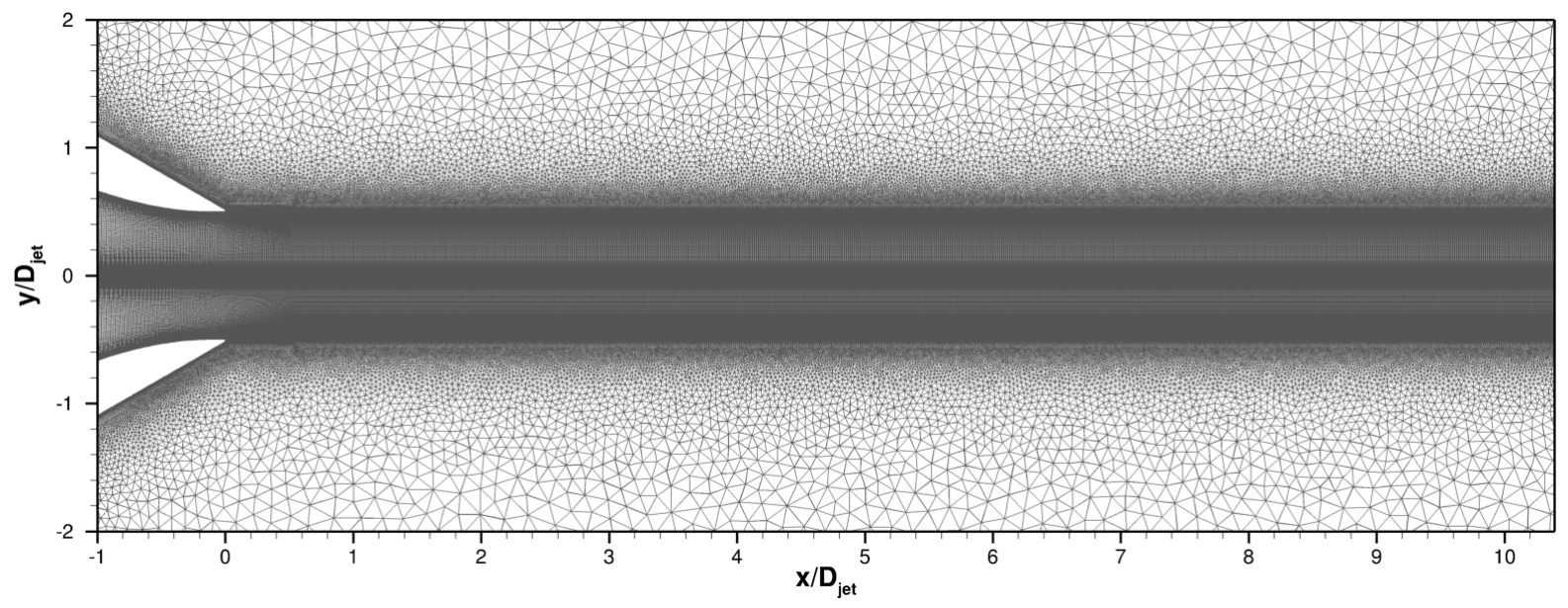

Figure 11.-The Case 40 grid near the nozzle exit, pictured along the symmetry plane. 


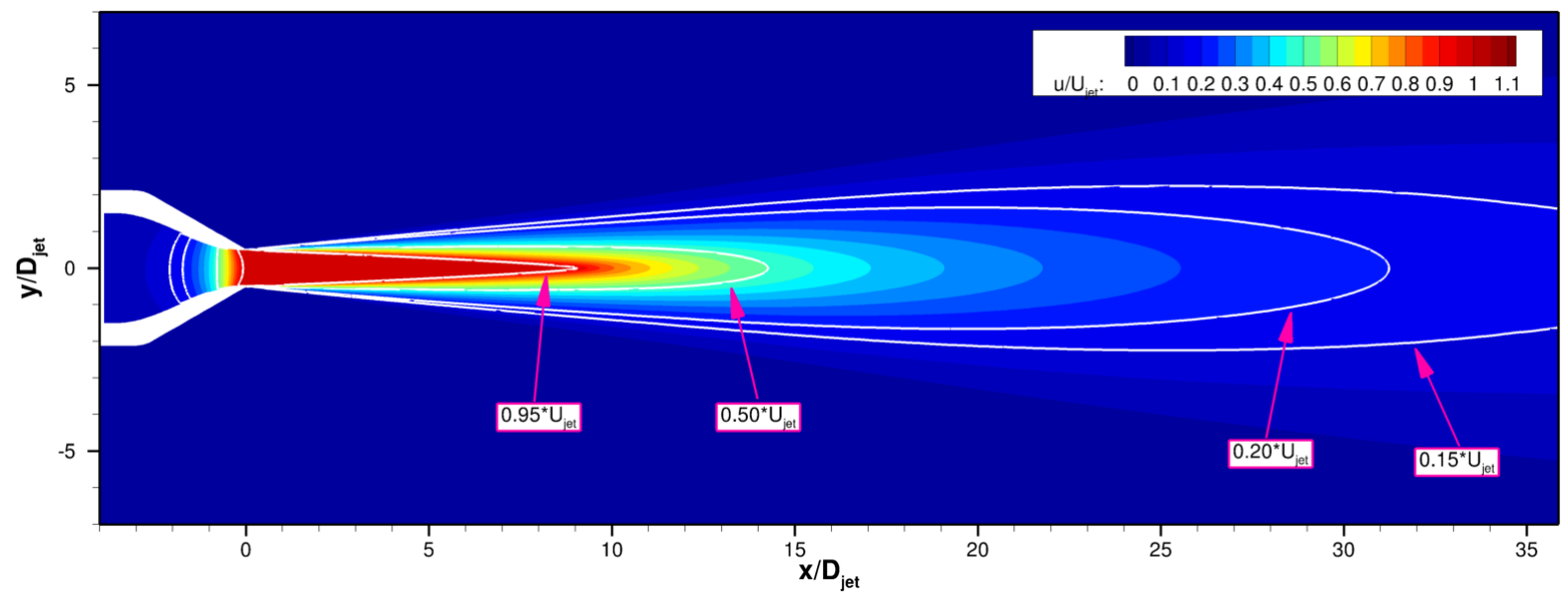

Figure 12.-Streamwise velocity, $u / U_{\text {jet }}$, contours of a FUN3D simulation using the Case $1 \mathrm{~b}$ grid with the SST-V turbulence model. White contour lines denote contour levels of 95, 50, 20, and 15 percent of $U_{\text {jet. }}$.

\subsection{Unstructured Grid Distributed Along Velocity Contours}

All the unstructured grids described above that included a region of refined grid in the jet plume used a block interface with a $1 \times D_{\text {jet }}$ diameter downstream of the nozzle exit for applying grid clustering. The Case 50 grid used the jet plume velocity contours to shape the jet plume refinement region. Figure 12 shows contours of streamwise velocity through the jet plume of the ARN2 flow from a FUN3D simulation using the Case $1 \mathrm{~b}$ grid and SST-V turbulence model. Contour lines are shown for 95, 50, 20, and 15 percent of $U_{\text {jet. }}$ In the Case 50 grid, a block interface was placed along the contour of 95 percent of $U_{j e t}$ in the jet plume. A second block interface was placed along the contour of 15 percent of $U_{j e t}$ in the jet plume from the nozzle exit to $30 \times D_{\text {jet }}$ downstream of the nozzle exit. Grid was clustered to these block interfaces: grid spacings of 0.03 in. along the surface and 0.02 in. radially. Additionally, a grid spacing of 0.03 in. was used along the jet centerline. The Case 50 grid was generated using only tetrahedral elements and consisted of 28.3 million nodes. Figure 13 shows two views of the Case 50 grid.

\subsection{Triangular Prism Grids}

The final set of grids, the Case $6 a$ and Case $6 \mathrm{~b}$ grids, consisted of triangular prism elements. The second finest 2D axisymmetric grid from the Turbulence Modeling Resource (Ref. 6) (with block sizes of $97 \times 97$ nodes inside the nozzle and $257 \times 225$ nodes downstream of the nozzle; used for Case 1a) was diagonalized using Pointwise ${ }^{\circledR}$. For the Case 6a grid, a diagonal was placed from each upstream lower corner node to the downstream upper corner node of each 2D cell, as pictured in Figure 14(a). The Case $6 \mathrm{~b}$ grid had diagonals placed from the upstream upper corner to the downstream lower corner of each 2D cell, as shown in Figure 14(b). As with Case 1a, the diagonalized 2D axisymmetric grids were then rotationally extruded 112 steps about the centerline to a $180^{\circ}$ sector. For the Case $6 \mathrm{a}$ and Case $6 \mathrm{~b}$ grids, the cells along the centerline were divided at a radius of 0.002 in. The outer cells were diagonalized and rotationally extruded with the rest of the grid. The cells adjacent to the centerline were replaced with triangular prism elements that were aligned with the centerline. This step was performed to eliminate zero-area cell faces when the 2D grid was extruded to a 3D grid. The Case $6 \mathrm{a}$ and Case $6 \mathrm{~b}$ grids are pictured in Figure 15. Each grid had 8.3 million nodes, only slightly more than the Case 1a grid. 


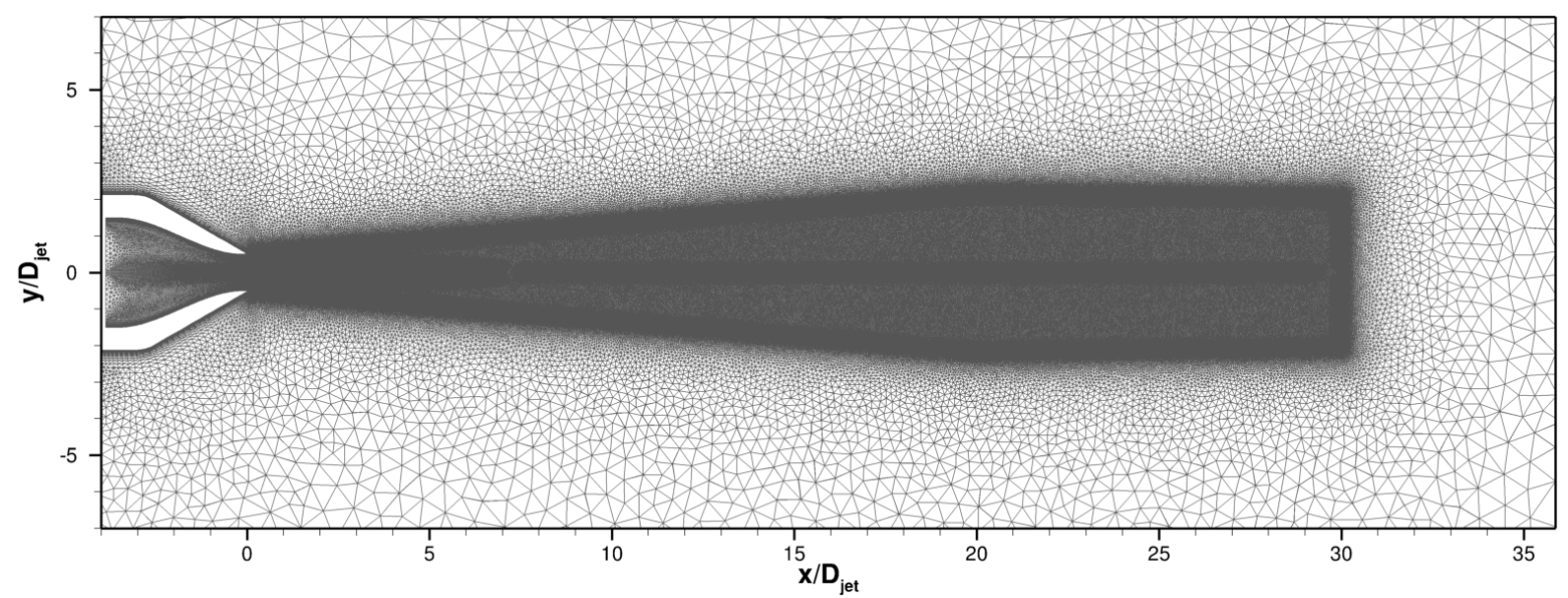

(a) Wide view.

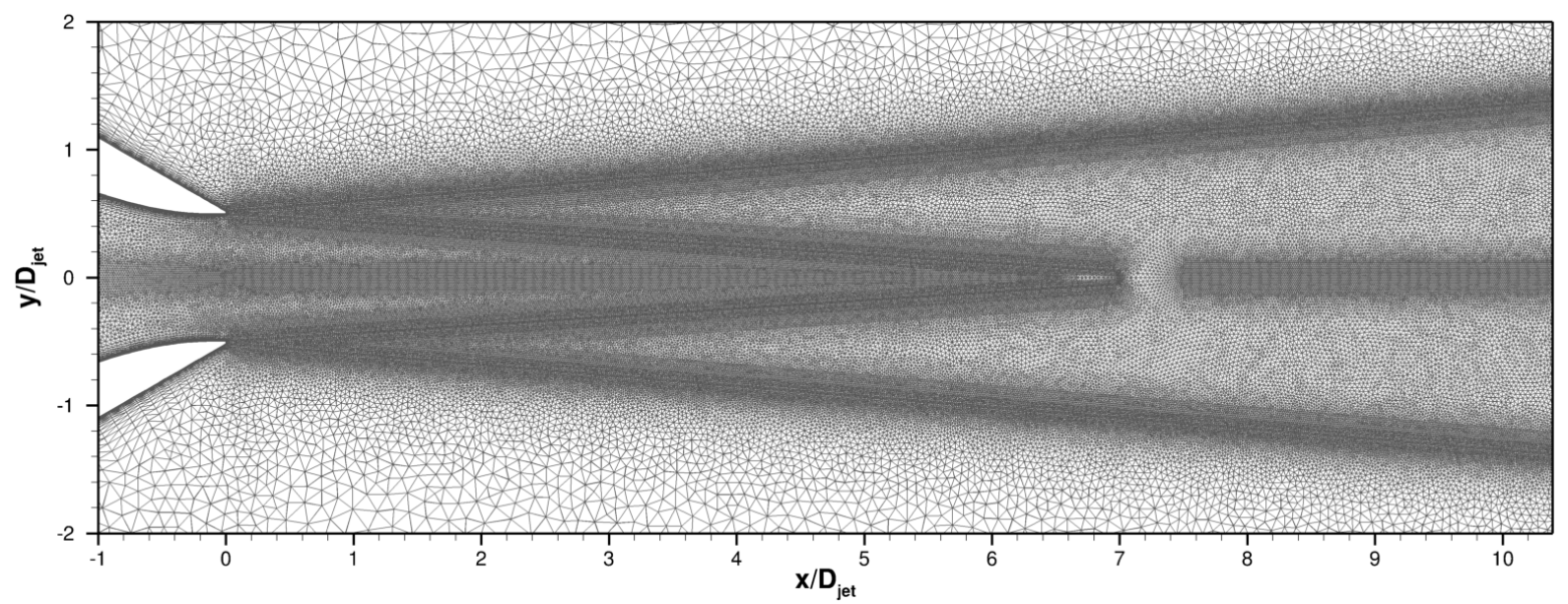

(b) View near nozzle exit.

Figure 13.-The Case 50 grid, pictured along the symmetry plane.

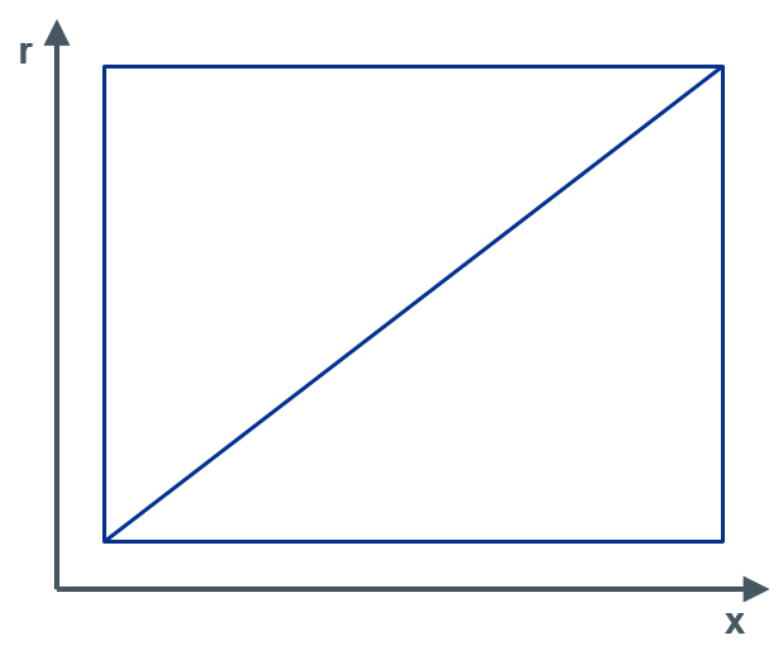

a) Case 6a.

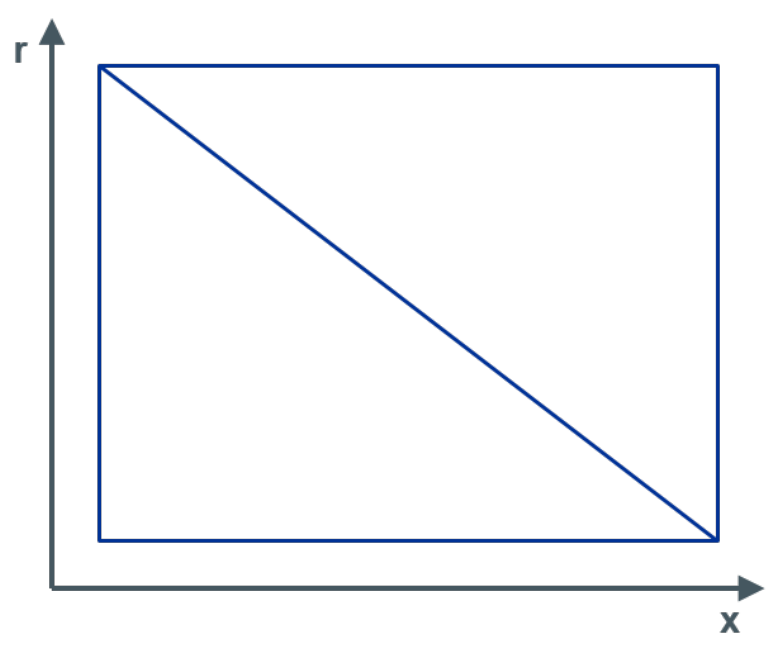

(b) Case 6b.

Figure 14.-Diagonalization of 2D axisymmetric grid cells for the Case $6 \mathrm{a}$ and Case $6 \mathrm{~b}$ grids. 


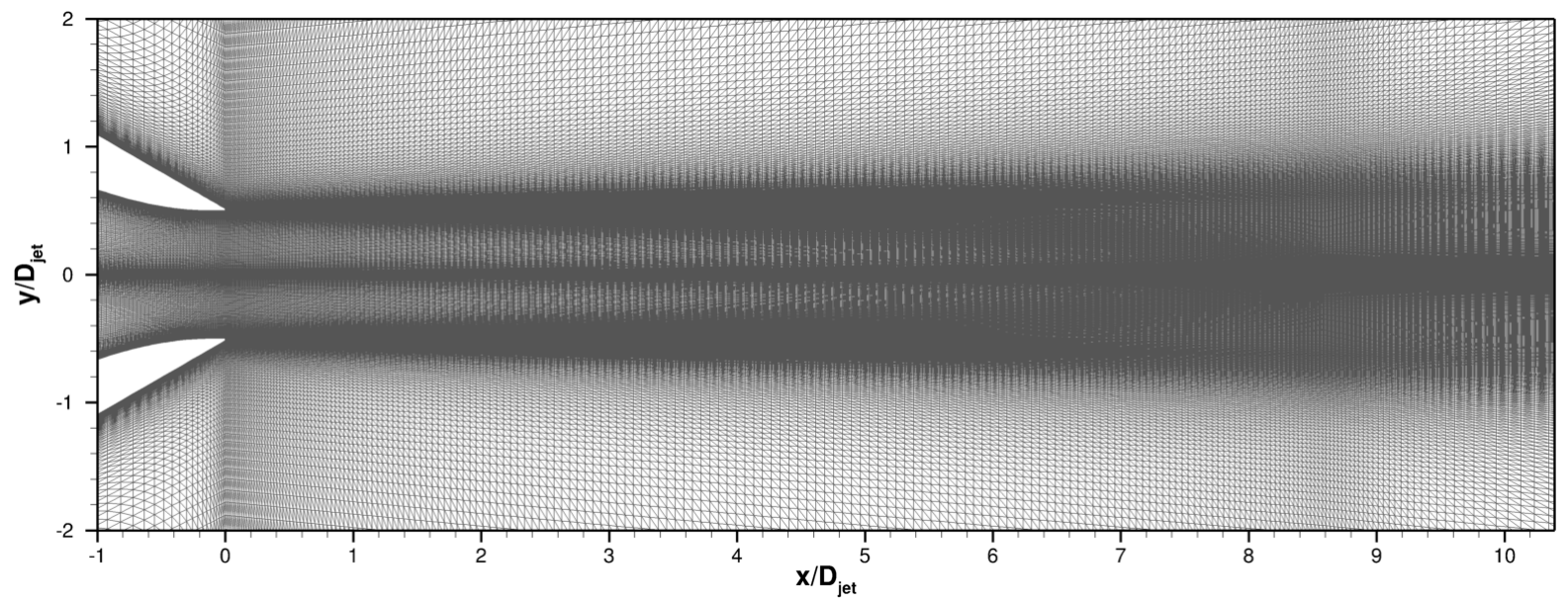

(a) Case 6a grid.

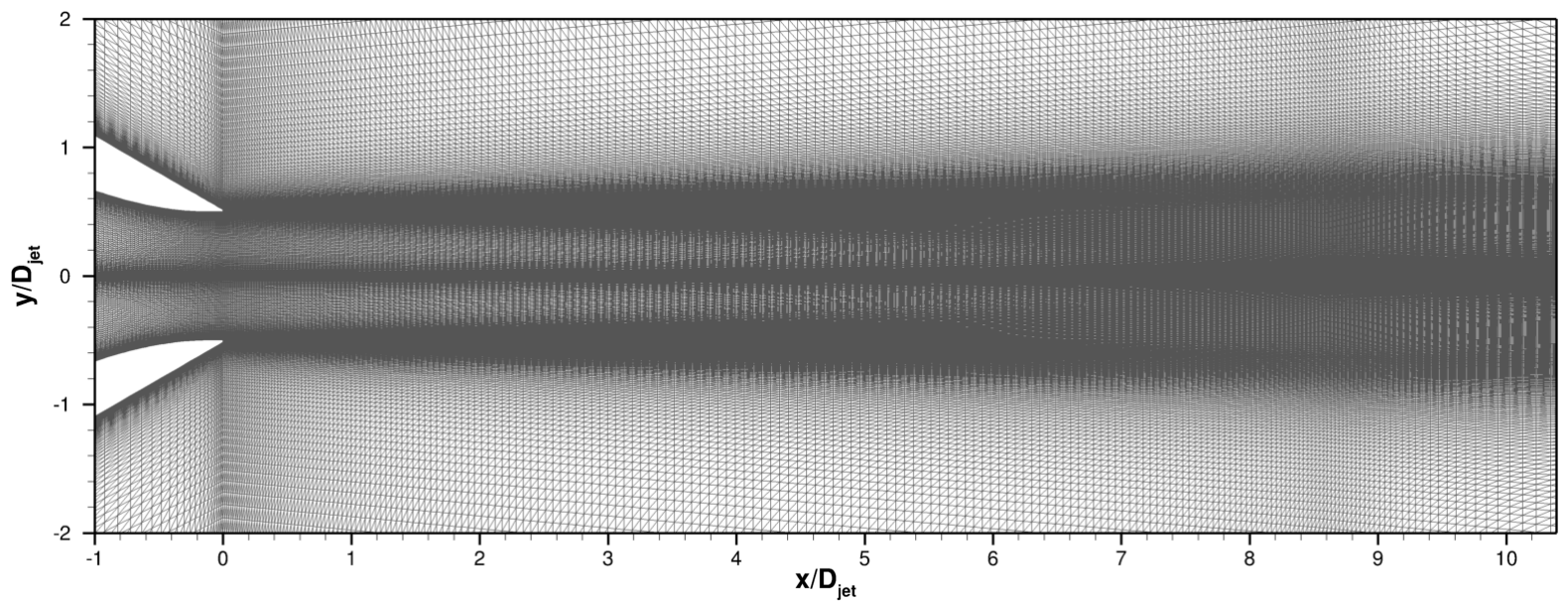

(b) Case 6b grid.

Figure 15.-The Case $6 \mathrm{a}$ and Case $6 \mathrm{~b}$ grids near the nozzle exit, pictured along the symmetry plane.

\subsection{Grid Comparisons}

There are several ways that the grids can be compared. The total number of nodes in each grid can be compared; these are listed in Table I. The grids range in size from 0.46 million nodes to 53.4 million nodes. Another way to compare the grids is by comparing their respective cell sizes. Figure 16 shows contours depicting the cell volume of the cells located near the symmetry plane of each grid. The plots for the unstructured grids (Case 20, Case 2a, Case 2d, Case 2j, Case 2k, Case 40, and Case 50) show how the grids were refined and how grid cells were clustered through each iteration of grid generation. As expected, the Case $2 \mathrm{k}$ grid has cells that are nearly an order of magnitude finer than the Case $2 \mathrm{j}$ grid. It is also interesting that the structured-like grids (Case 1a, Case 1b, Case 1c, Case 6a, and Case 6b) have larger cells in the jet plume region than many of the unstructured grids. 


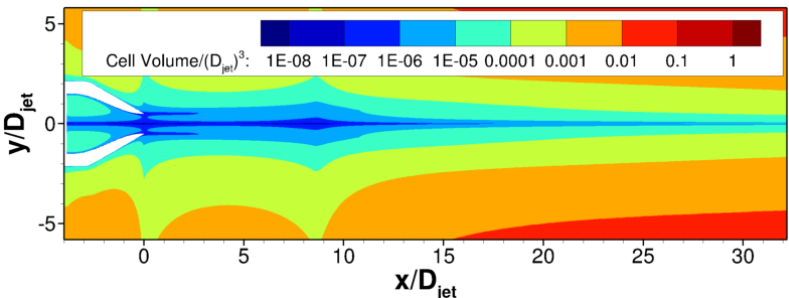

(a) Case 1a grid.

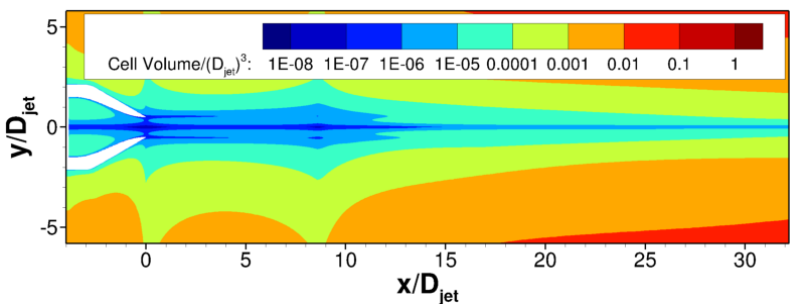

b) Case $1 \mathrm{~b}$ grid.

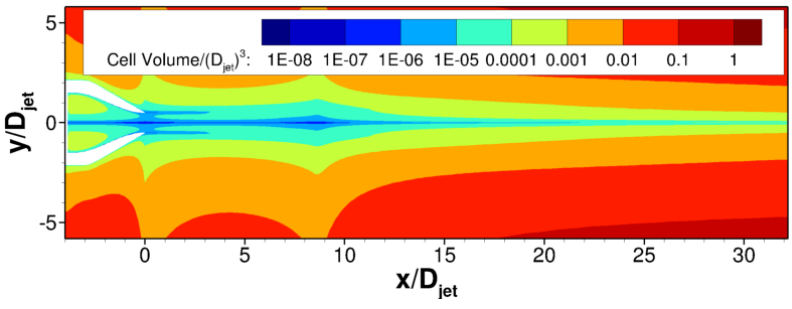

(c) Case 1c grid.

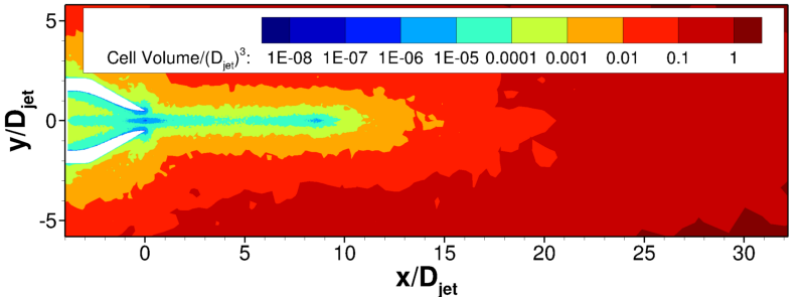

(d) Case 20 grid.

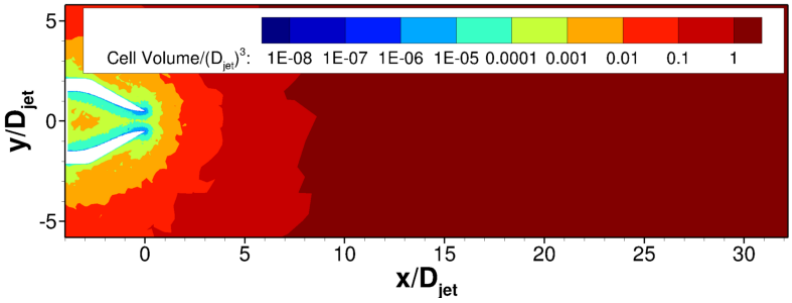

(e) Case 2a grid.

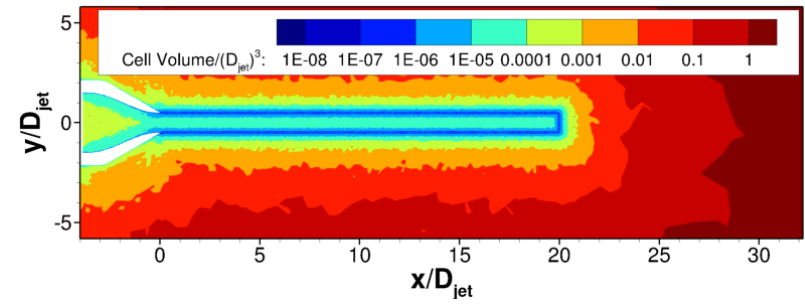

(f) Case 2d grid.

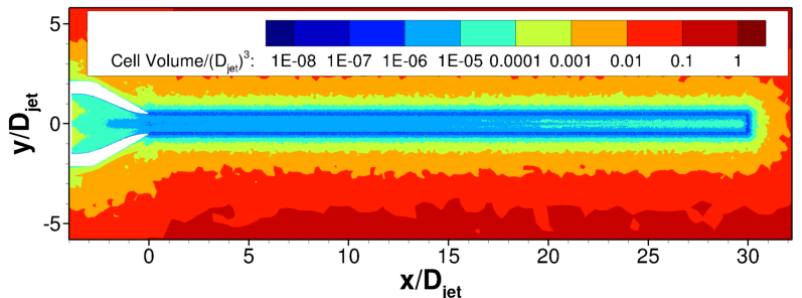

(g) Case 2j grid.

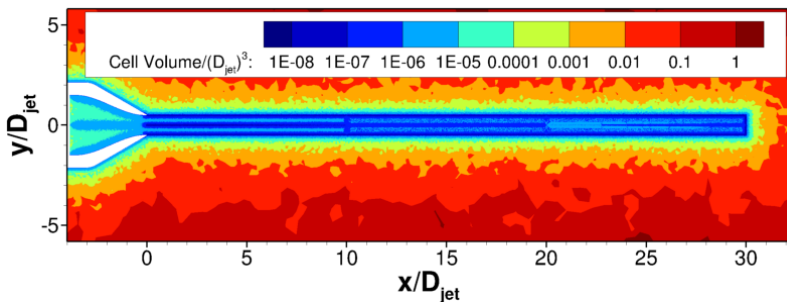

(h) Case 2k grid.

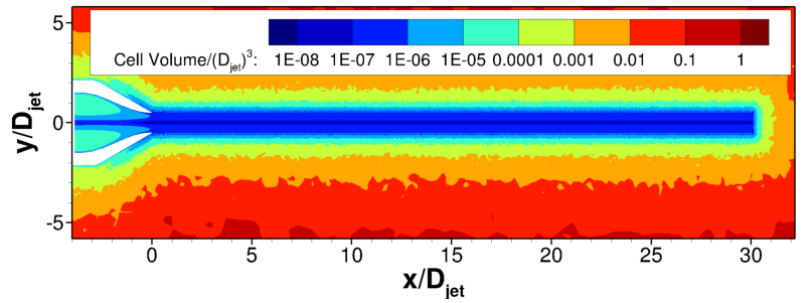

(i) Case 40 grid.

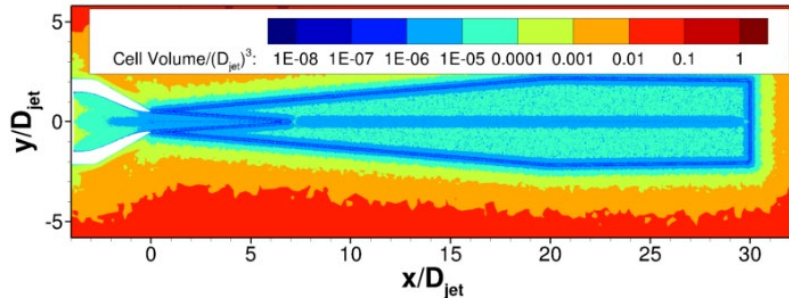

(j) Case 50 grid.

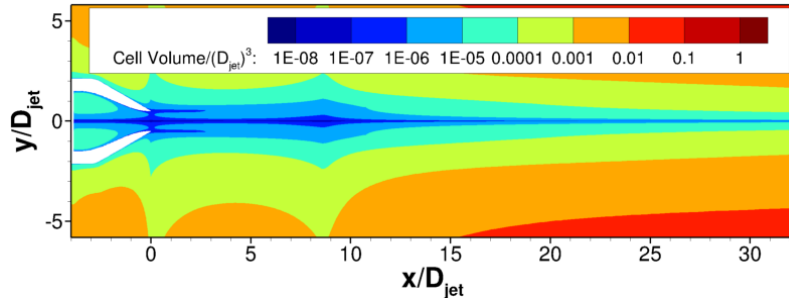

(k) Case 6a grid.

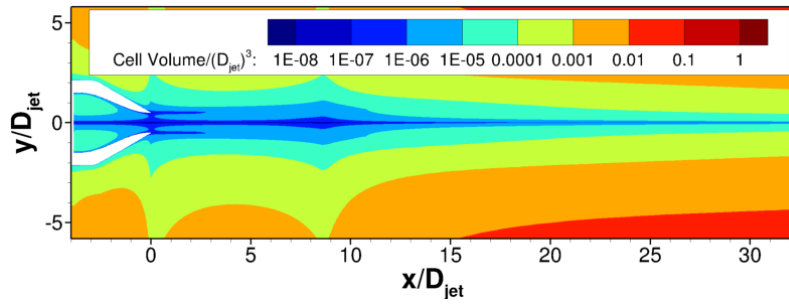

(I) Case 6b grid.

Figure 16.-Contours showing cell volume of cells near the symmetry plane for selected grids. 


\subsection{Results}

The results of the RANS simulations with the grids described earlier will be discussed here. The simulations were performed using FUN3D. Flow simulations were completed for all the grids using Menter's Shear Stress Transport (SST-V) turbulence model; these will be presented first. Simulations were also performed using the Spalart-Allmaras (S-A) and $k-k L$ turbulence models on several select grids; these results will be discussed later. As was stated earlier, it is assumed that, for a given turbulence model, grid-converged solutions should be similar for RANS simulations.

\subsection{Structured-Like Grids}

The structured-like grids - Case 1a, Case 1b, and Case 1c - are the first solutions to be presented. Figure 17 shows the nondimensionalized streamwise velocity, $u / U_{j e t}$, and turbulent kinetic energy, $k /\left(U_{j e t}\right)^{2}$, along the jet centerline for Case 1a, Case 1b, and Case 1c. The solutions are compared to the experimental data and the baseline structured grid simulation (Wind-US). The Case 1a, Case 1b, and Case 1c solutions all show excellent agreement with the Wind-US baseline solution for both the centerline streamwise velocity and turbulent kinetic energy. The coarsened grid (Case 1c) shows good agreement with the fine grids (Case 1a and Case 1b) despite the grid having roughly 87 percent fewer nodes. The initial jet plume decay of the coarsened grid (Case 1c) moves upstream about $0.3 \times D_{\text {jet }}$, but the rate of jet plume decay and peak level of turbulent kinetic energy (and its location) agree with the fine grids (Case 1a and Case 1b). These three simulations show that FUN3D can produce a nearly identical solution to the baseline Wind-US structured grid solution — and other structured-grid RANS solver flow solutions, including CFL3D and OVERFLOW-using a structured-like grid, and that this solution is grid-converged.

\subsection{First Series of Unstructured Grids}

Simulations of the first set of fully tetrahedral, unstructured grids (Case 20 and Case 2a through Case 2d) were completed next. Simulations were also performed on the Case 30 and Case 3a through Case $3 \mathrm{~d}$ grids, since they are essentially the same grids as Case 20 and Case 2a through Case 2d, but were exported from Pointwise ${ }^{\circledR}$ using the Combine Anisotropic Tetrahedra option. The streamwise velocity and turbulent kinetic energy along the centerline are plotted in Figure 18. The plots show that there is good
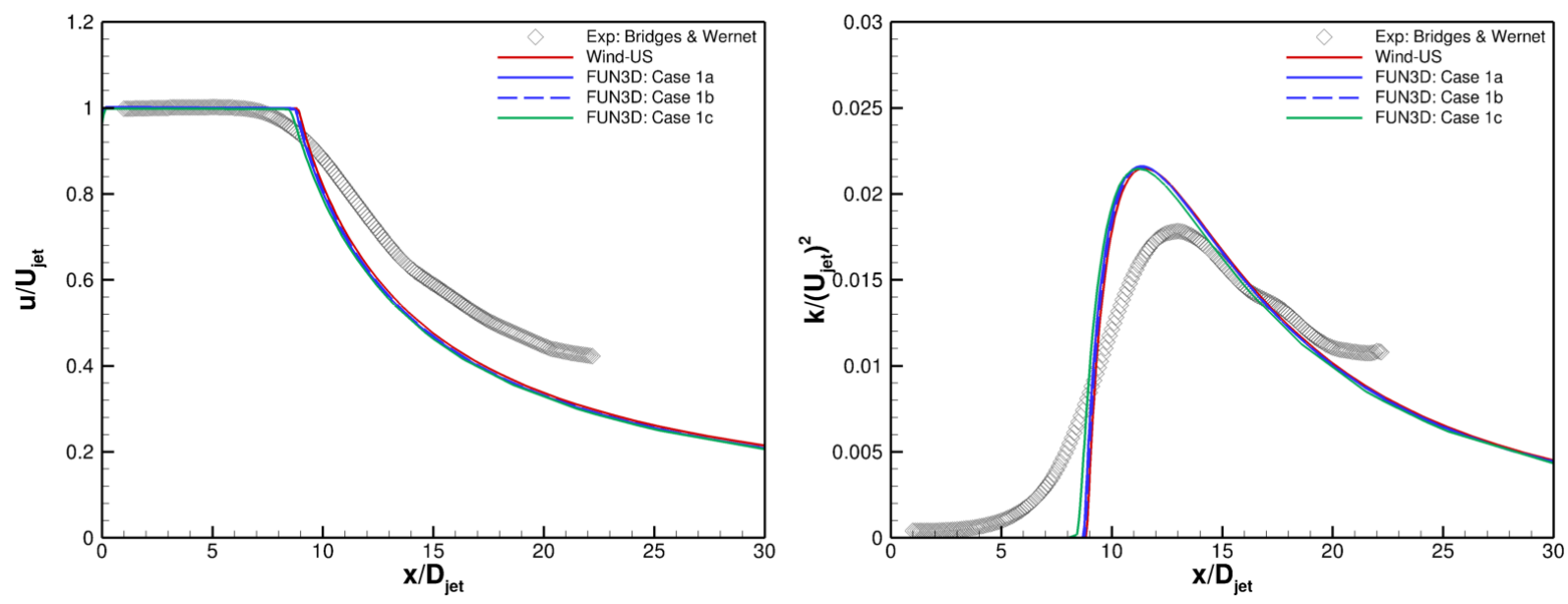

Figure 17.-Comparison of streamwise velocity, $u / U_{j e t}$, and turbulent kinetic energy, $k /\left(U_{j e t}\right)^{2}$, along the centerline for experimental data and RANS solutions using the SST-V turbulence model. 

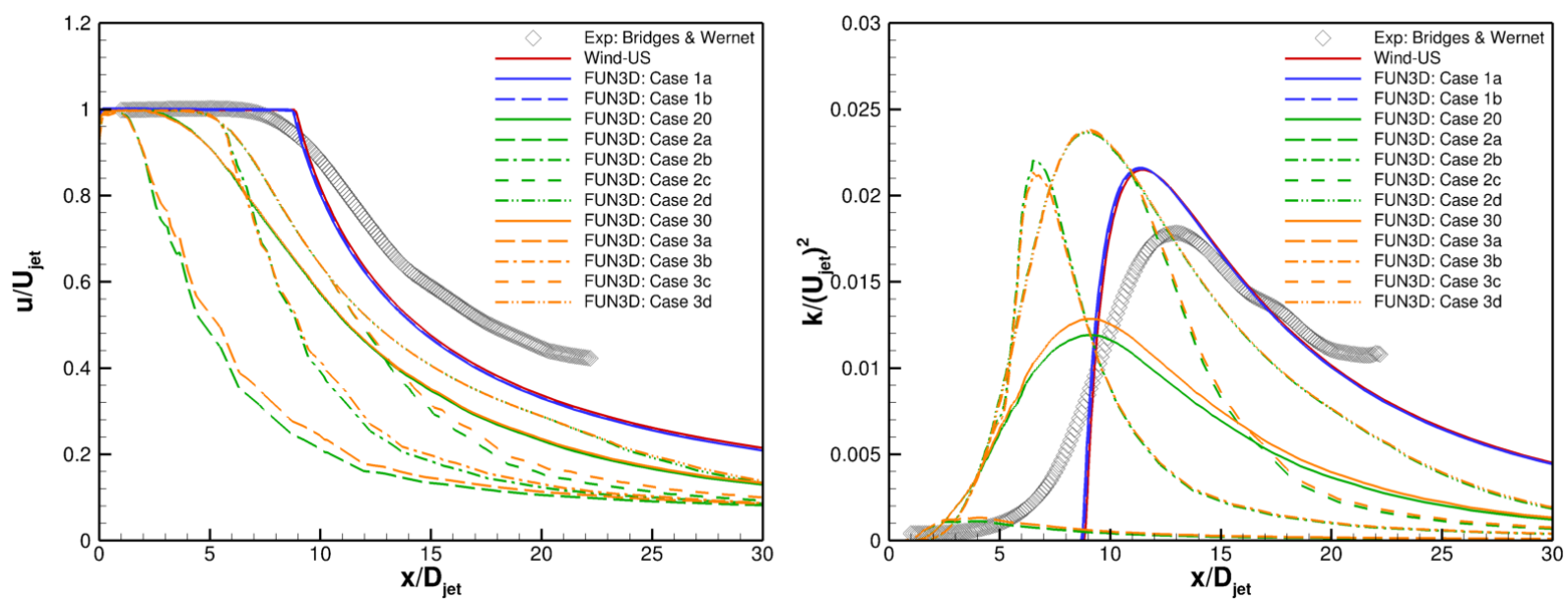

Figure 18.-Comparison of streamwise velocity, $u / U_{j e t}$, and turbulent kinetic energy, $k /\left(U_{j e t}\right)^{2}$, along the centerline for experimental data and RANS solutions using the SST-V turbulence model.

agreement between each fully tetrahedral grid solution and its corresponding combined anisotropic tetrahedral grid solution (e.g., Case 2a and Case 3a). This is not unexpected, as FUN3D is a node-based flow solver and each pair of grids have the same grid node topology; only the grid cells are different in the areas where they have been combined. Since the solutions between each grid pair are so similar for FUN3D, the Combined Anisotropic Tetrahedra option was not used on subsequent grids. It should be noted that the Case may be different for a cell-based flow solver, but this was not tested in this study.

The more important result to note from Figure 18 is that the unstructured grid simulations all produce significantly shorter jet plumes than the baseline solution. Looking at the plots of streamwise velocity along the centerline, there is a noticeable trend for Case 2a through Case 2d (and also Case 3a through Case $3 d$ ) in which the jet plume also lengthens (from $1.03 \times D_{\text {jet }}$ to $4.68 \times D_{j e t}$ ) as the length of the grid refinement region lengthens (from $0 \times D_{j e t}$ to $20 \times D_{j e t}$ ). The baseline SST-V solution predicts the jet plume to begin decaying $8.93 \times D_{j e t}$ downstream of the nozzle exit. Therefore, even the best tetrahedral solution (Case $2 \mathrm{~d}$ ) predicts the jet plume to be about $4.25 \times D_{\text {jet }}$ shorter than the baseline. Case $2 \mathrm{~b}$ through Case $2 \mathrm{~d}$ predict the jet plume to begin decaying at about the same location, but the rate of decay varies with the length of the jet plume grid refinement region.

The plots of turbulent kinetic energy of Case 2a through Case 2d show the fully tetrahedral cases to behave much differently than the baseline structured grid simulation. The peak levels of turbulent kinetic energy of Case 2a through Case $2 \mathrm{~d}$ are all found much earlier $\left(2.6 \times D_{\text {jet }}\right.$ to $7.5 \times D_{\text {jet }}$ earlier $)$ than the baseline structured grid solution $\left(11.55 \times D_{j e t}\right)$, causing the shorter jet plumes. For Case $2 \mathrm{~b}$ through Case $2 \mathrm{~d}$, the earlier jet plume decay also results in a larger peak turbulent kinetic energy, $k /\left(U_{j e t}\right)^{2}=0.022$ to 0.024 , than the baseline structured grid solution, $k /\left(U_{j e t}\right)^{2}=0.021$.

The Case 20 solution is quite a bit different than the Case 2a through Case $2 \mathrm{~d}$ solutions, which is likely the result of having a different grid topology in the jet plume (grid refinement only along the centerline rather than through the entire diameter of the plume). The jet plume of Case 20 begins decaying about $4.03 \times D_{\text {jet }}$ downstream of the nozzle exit. The rate of jet plume decay is initially less steep than the baseline structured grid solution, but eventually takes on a similar rate of decay by the time $u / U_{j e t}=0.6$. Unlike Case $2 \mathrm{~b}$ through Case 2d, the peak turbulent kinetic energy along the jet centerline of Case 20, $k /\left(U_{j e t}\right)^{2}=0.011$, is significantly less than that of the baseline structured grid case. However, the peak level of turbulent kinetic energy along the centerline takes place at about the same location as in Case 2c and Case $2 \mathrm{~d}, x / D_{j e t}=9.1$; this is still upstream of the location observed in the baseline Wind-US solution. 

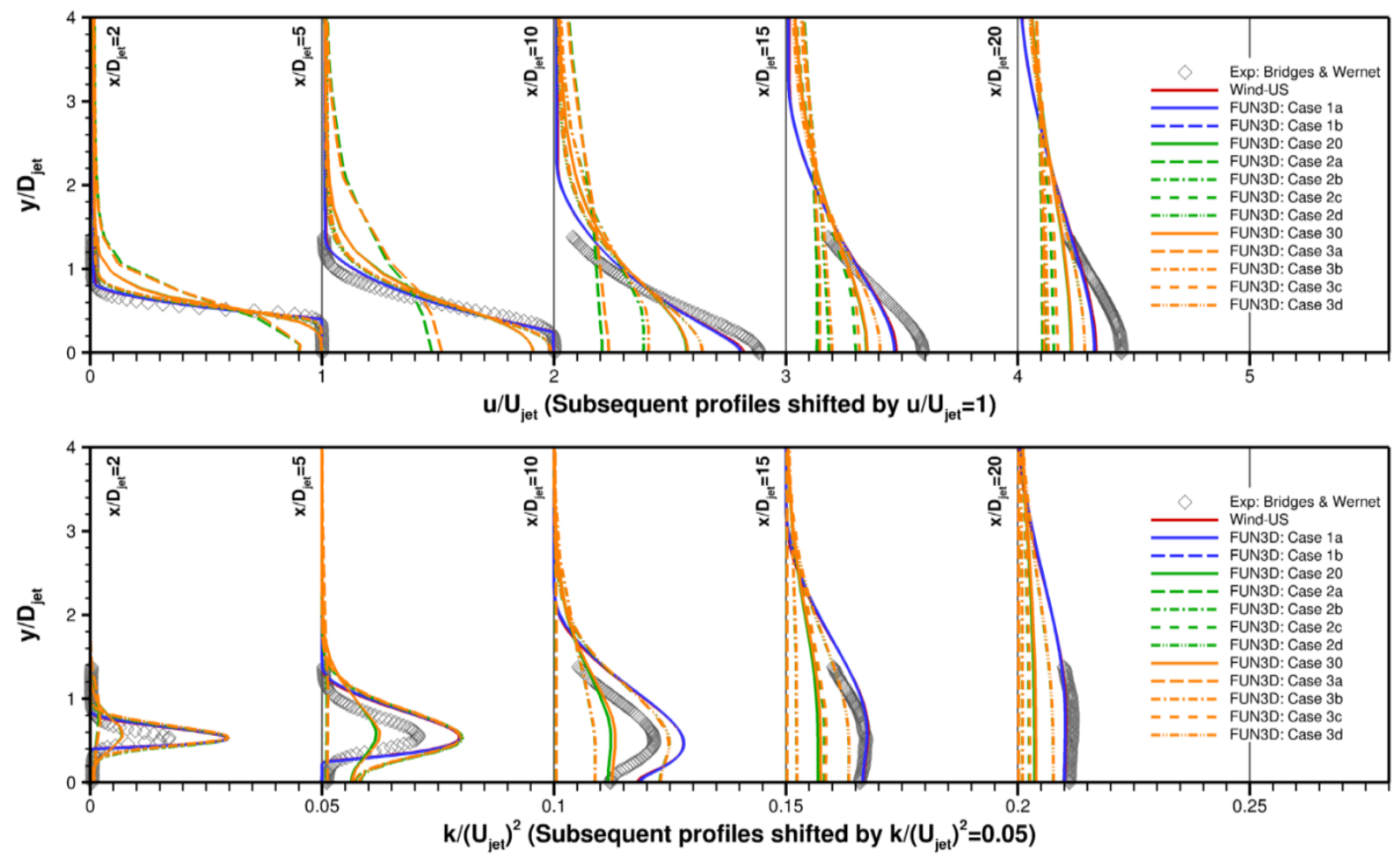

Figure 19.-Comparison of streamwise velocity, $u / U_{\text {jet }}$, (top) and turbulent kinetic energy, $k /\left(U_{j e t}\right)^{2}$, (bottom) at stations through the jet plume for the experimental data and RANS solutions using the SST-V turbulence model.

Figure 19 shows plots of nondimensionalized streamwise velocity and turbulent kinetic energy radially through the jet plume at five streamwise locations. Most likely due to the lack of grid refinement in the shear layer, the Case 20 grid solution consistently underpredicts the peak value of turbulent kinetic energy at all streamwise locations in the shear layer $\left(y / D_{j e t}=0.5\right)$. Therefore, future grids need refinement in the shear layer.

Continuing to consider Figure 19, the Case 2c and Case 2d grid solutions show good agreement with the baseline structured grid solution's peak turbulent kinetic energy in the shear layer up to $5 \times D_{j e t}$ downstream of the nozzle exit; however, the unstructured solutions overpredict turbulent kinetic energy along the centerline at these locations. The Case 2a through Case $2 \mathrm{~d}$ grids lack grid refinement along the centerline; therefore, future grids also need refinement along the jet plume centerline.

\subsection{Second Series of Unstructured Grids}

The Case 2e through Case 2j grids followed the lessons learned from the simulations of Case 20 and Case 2a through Case 2d and applied greater grid refinement to the centerline and shear layer of the jet plume. Plots of streamwise velocity and turbulent kinetic energy along the jet centerline are shown in Figure 20. The Case 2e through Case $2 \mathrm{j}$ grid simulations predict a slightly later jet plume decay than Case $2 \mathrm{~d}\left(5.28 \times D_{j e t}\right.$ to $5.47 \times D_{\text {jet }}$ vs. $\left.4.68 \times D_{j e t}\right)$, but the jet plume decay is still much earlier than the baseline structured grid solution $\left(8.93 \times D_{j e t}\right)$. The peak turbulent kinetic energy along the centerline of the Case 2e through Case 2j solutions all agree with each other $\left(k /\left(U_{j e t}\right)^{2}=0.023\right.$ at $x / D_{j e t}=9.2$, but the peak is greater and occurs earlier than the baseline structured grid solution $\left(k /\left(U_{j e t}\right)^{2}=0.021\right.$ at $\left.x / D_{j e t}=11.55\right)$. It is particularly interesting to note that for the Case 2e through Case $2 \mathrm{j}$ grids, the solutions seem to converge upon a common solution (best seen by the Case $2 \mathrm{i}$ and Case $2 \mathrm{j}$ solutions), but the jet plume decays rapidly beyond the jet plume refinement region (e.g., $10 \times D_{j e t}$ for Case 2e, $15 \times D_{j e t}$ for Case $2 \mathrm{f}$, etc.). Despite the 

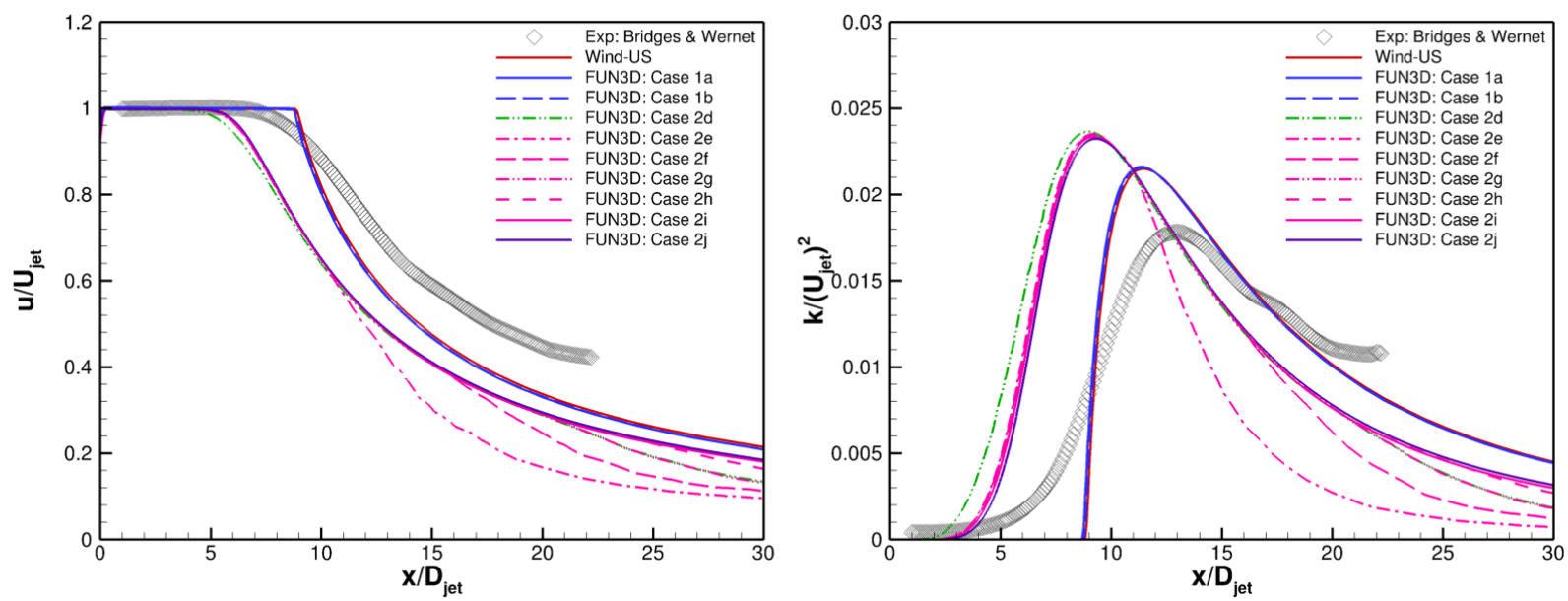

Figure 20.-Comparison of streamwise velocity, $u / U_{\text {jet }}$, and turbulent kinetic energy, $k /\left(U_{j e t}\right)^{2}$, along the centerline for experimental data and RANS solutions using the SST-V turbulence model.

Case $2 \mathrm{i}$ and Case $2 \mathrm{j}$ grids using different algorithms to generate the surface grids (Delaunay and Advancing Front, respectively), there is good agreement between the two solutions.

\subsection{Extra Fine Unstructured Grid}

The Case $2 \mathrm{k}$ grid had 10 times the number of grid points as the finest of the previous fully tetrahedral unstructured grids (53.4 million nodes vs. 5.3 million nodes). It was assumed that increased grid resolution would help the solution agree more closely with the baseline structured grid solution. The plots of streamwise velocity and turbulent kinetic energy along the centerline are shown in Figure 21 . The Case $2 \mathrm{k}$ solution predicts the jet plume decay to begin $5.75 \times D_{\text {jet }}$ downstream of the nozzle exit, which is slightly further downstream than the $5.47 \times D_{\text {jet }}$ observed in the Case $2 \mathrm{j}$ solution, but still quite earlier than the $8.93 \times D_{\text {jet }}$ observed in the baseline structured grid solution. Surprisingly, the rate of decay following the initial decay of the jet plume is much steeper than that of the Case $2 \mathrm{j}$ solution. By $20 \times D_{\text {jet }}$ downstream of the nozzle exit, the Case $2 \mathrm{k}$ solution is predicting the velocity at the centerline to be only $0.14 \times U_{j e t}$, compared to $0.29 \times U_{\text {jet }}$ for the Case $2 \mathrm{j}$ solution and $0.33 \times U_{\text {jet }}$ for the baseline Wind-US solution.

Consequently, the increased rate of jet plume decay in Case $2 \mathrm{k}$ results in a larger peak turbulent kinetic energy along the centerline $\left(k /\left(U_{j e t}\right)^{2}=0.026\right)$ than what is observed in Case $2 \mathrm{j}$.

The overall structure of the turbulent kinetic energy field within the jet plume was unexpectedly different for the Case $2 \mathrm{k}$ grid simulation. Contours of turbulent kinetic energy are compared for the Case $1 \mathrm{~b}$ grid, Case $2 \mathrm{j}$ grid, and Case $2 \mathrm{k}$ grid simulations in Figure 22. (The Case $1 \mathrm{~b}$ grid solution is used since it is very similar to the baseline structured grid solution.) As observed in Figure 22(c), the turbulent kinetic energy field of the Case $2 \mathrm{k}$ grid simulation is considerably shorter along the centerline than that of the Case $1 \mathrm{~b}$ or Case $2 \mathrm{j}$ grid simulations. Whereas the Case $2 \mathrm{k}$ grid simulation has a greater peak turbulent kinetic energy along the centerline, the regions of high turbulent kinetic energy along the shear layer are not as intense as those in the Case $1 \mathrm{~b}$ and Case $2 \mathrm{j}$ grid solutions $\left(k /\left(U_{\text {jet }}\right)^{2}=0.026 \mathrm{vs.} k /\left(U_{\text {jet }}\right)^{2}=0.030\right)$. Lastly, the turbulent kinetic energy field of the Case $2 \mathrm{k}$ grid simulation has two noticeable qualitative differences from those of the Case $1 \mathrm{~b}$ and Case $2 \mathrm{j}$ grid simulations: 1 ) it is less full in the radial direction between $x / D_{\text {jet }}=8$ and $x / D_{\text {jet }}=11$; and 2 ) it is less smooth overall, appearing as if the solution was not fully converged despite all indicators showing the Case $2 \mathrm{k}$ grid simulation had converged. The solution from the Case $2 \mathrm{k}$ grid defied expectations (that a finer grid would produce a better solution) and demonstrated that simply increasing grid resolution without concern for the type of grid cell may not produce a solution that more closely agrees with the baseline structured grid solution. 

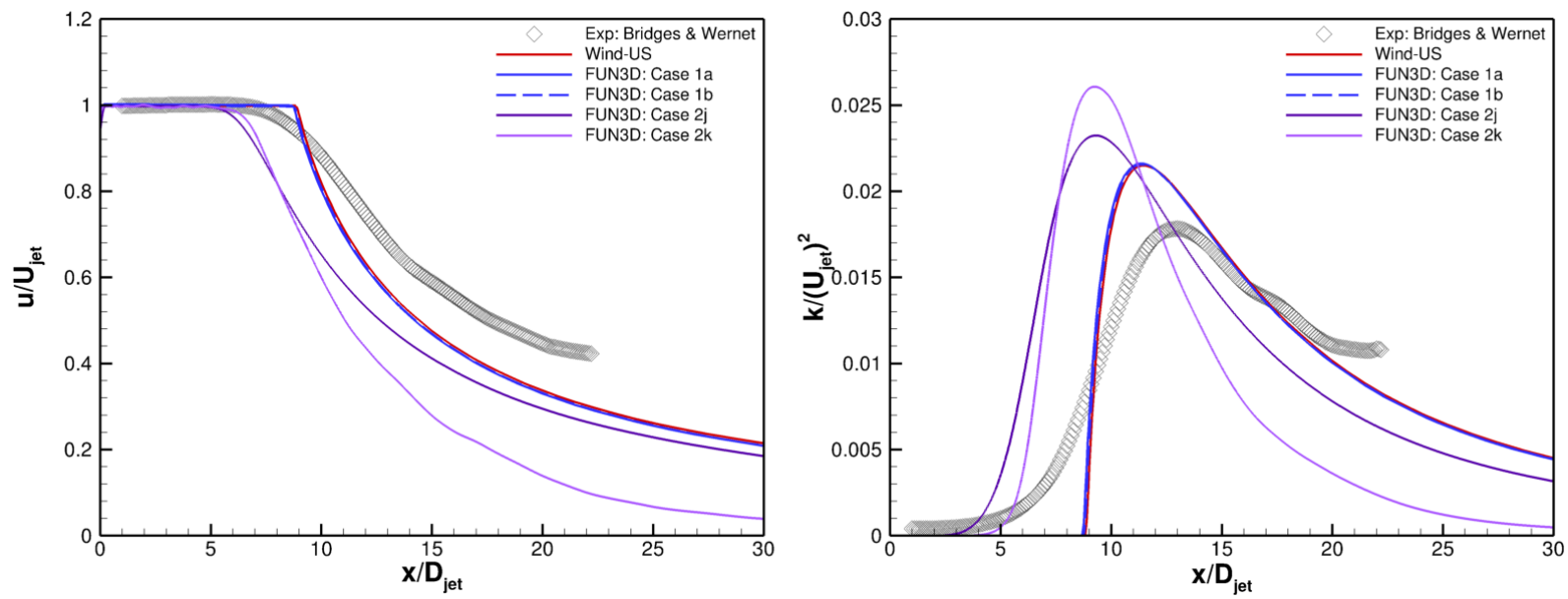

Figure 21.-Comparison of streamwise velocity, $u / \bigcup_{j e t}$, and turbulent kinetic energy, $k /\left(U_{j e t}\right)^{2}$, along the centerline for experimental data and RANS solutions using the SST-V turbulence model.

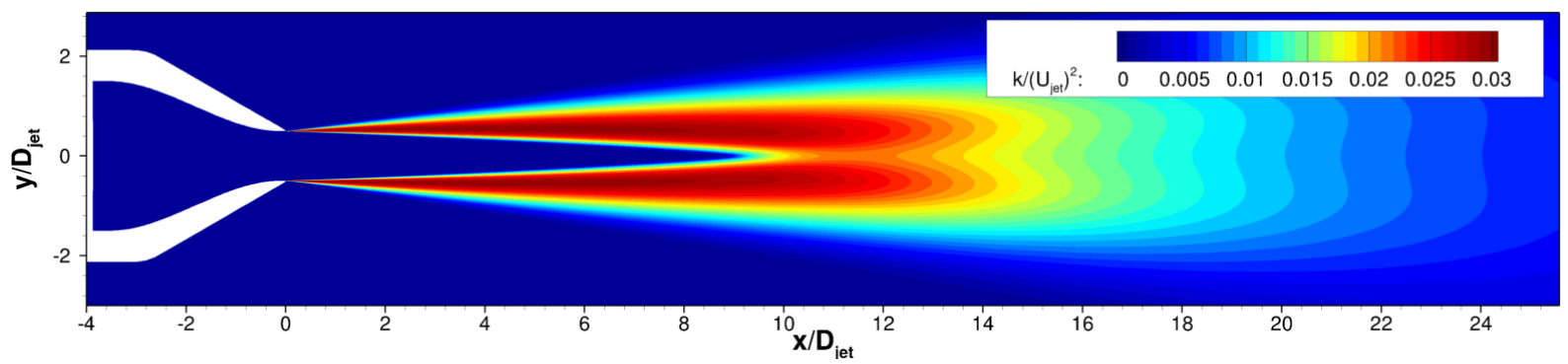

a) Case 1b.

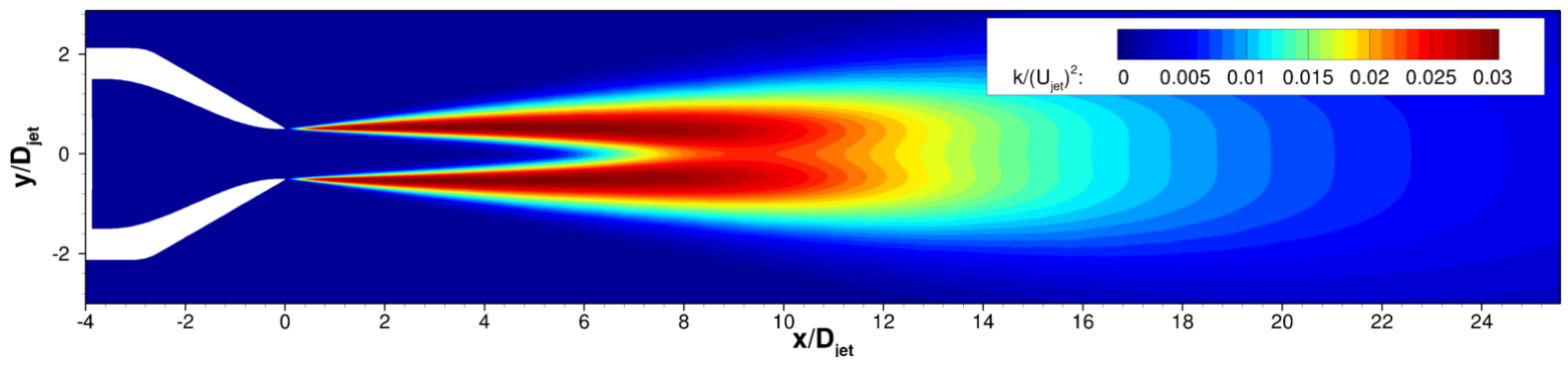

b) Case $2 \mathbf{j}$.

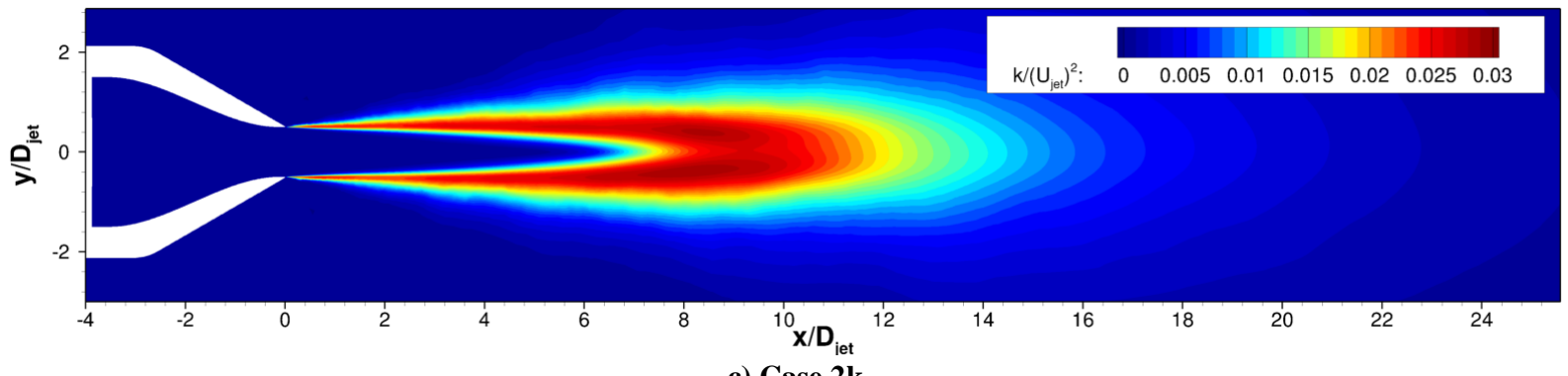

c) Case 2k.

Figure 22.-Contours of turbulent kinetic energy, $k /\left(U_{j e t}\right)^{2}$, along symmetry plane for selected RANS simulations using structured and unstructured grids and SST-V turbulence model. 


\subsection{Hybrid Grid}

The Case 40 grid was an attempt to solve the ARN2 jet flow with a hybrid grid, with structured-like elements in the jet plume and unstructured tetrahedral elements in the surrounding flowfield. In Figure 23, the centerline streamwise velocity and turbulent kinetic energy are plotted. The Case 40 grid solution shows good agreement with the baseline structured grid solution. The plume decay is only about $0.015 \times D_{\text {jet }}$ later than the baseline Wind-US solution. Subsequently, the peak turbulent kinetic energy along the centerline is 1.5 percent less than that of the baseline Wind-US solution and decays at a slightly faster rate through the rest of the jet plume.

Looking at radial profiles of streamwise velocity and turbulent kinetic energy plotted at stations through the jet plume in Figure 24, the Case 40 grid solution continues to show excellent agreement with the baseline Wind-US solution through the entire plume, and notably in the shear layer where other unstructured grid simulations showed less than favorable agreement. The Case 40 grid simulation, using a hybrid grid with structured-like elements in the jet plume region, hints that a structured-like grid in the jet plume produces the best agreement with the baseline solution.

\subsection{Unstructured Grid Distributed Along Velocity Contours}

The Case 50 grid applied unstructured grid refinement along several velocity contours of a known solution (i.e., the structured Case $1 \mathrm{~b}$ grid solution). The centerline velocity and turbulent kinetic energy of the Case 50 grid simulation are plotted and compared to previous solutions in Figure 25. The centerline velocity of the Case 50 grid solution closely agrees with the Case $2 \mathrm{j}$ solution to about $10 \times D_{\text {jet }}$, after which the jet plume decays at a slower rate. The Case 50 grid peak turbulent kinetic energy along the centerline agrees with the Case $2 \mathrm{j}$ grid solution in magnitude and location, however the rise and decay of the turbulent kinetic energy is somewhat later. Despite the attempt to use a known solution to determine refinement within an unstructured grid, the Case 50 grid solution was still no closer to the baseline solution than the previous unstructured grid solutions.
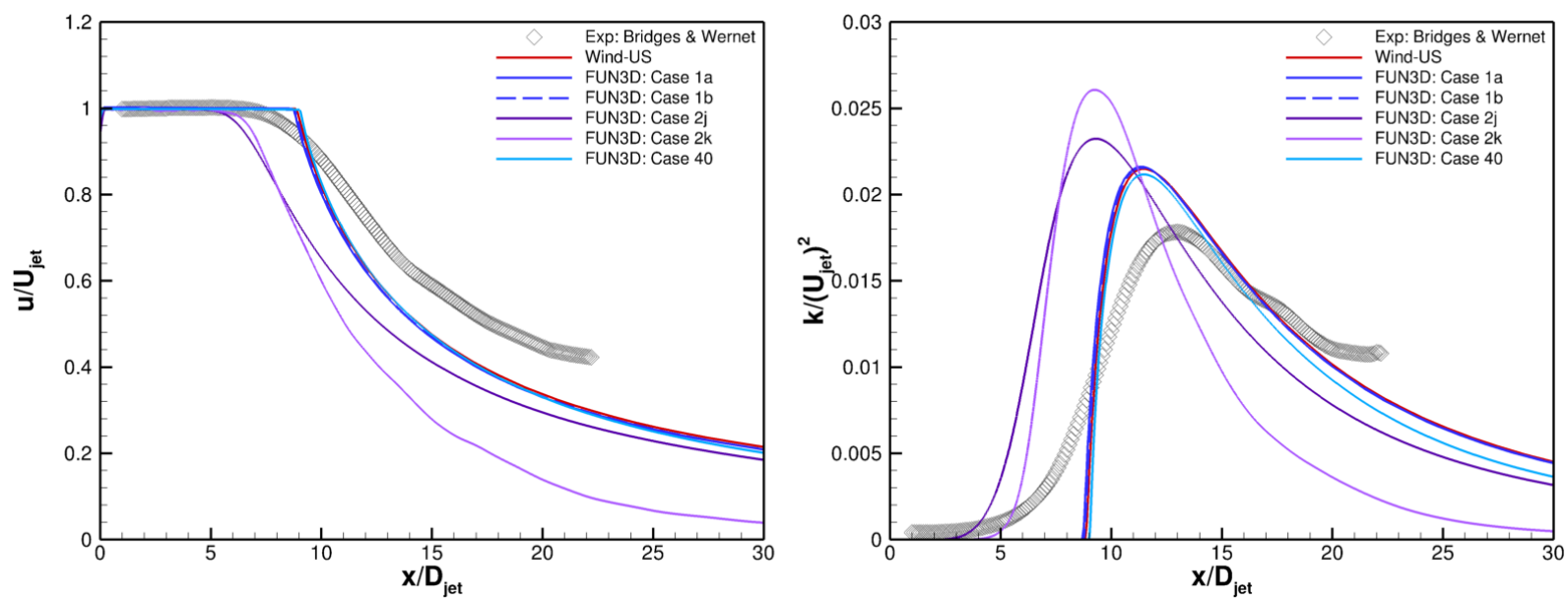

Figure 23.-Comparison of streamwise velocity, $u / U_{\text {jet }}$, and turbulent kinetic energy, $k /\left(U_{\text {jet }}\right)^{2}$, along the centerline for experimental data and RANS solutions using the SST-V turbulence model. 

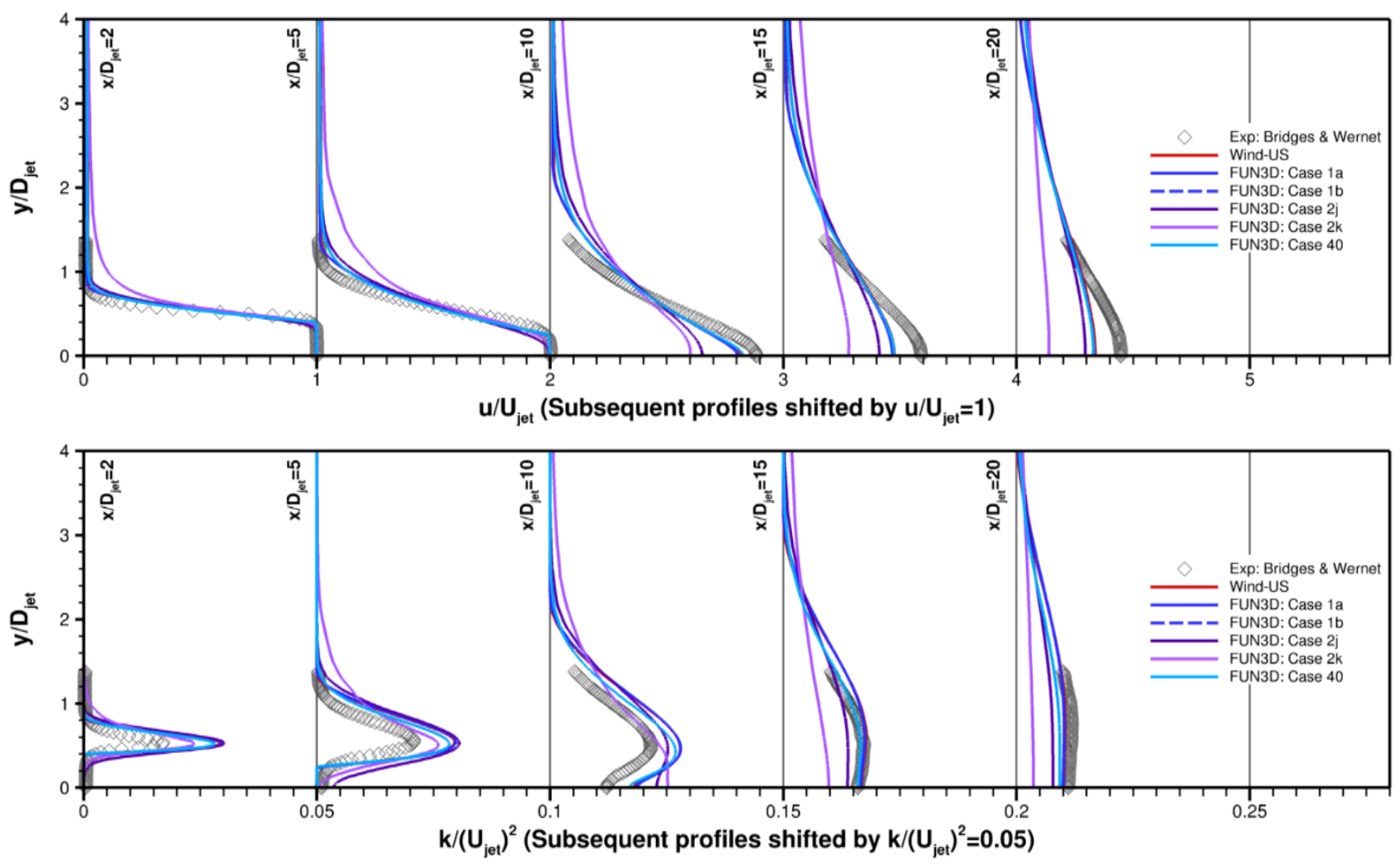

Figure 24.-Comparison of streamwise velocity, $u / \bigcup_{j e t}$, (top) and turbulent kinetic energy, $k /\left(U_{j e t}\right)^{2}$, (bottom) at stations through the jet plume for the experimental data and RANS solutions using the SST-V turbulence model.
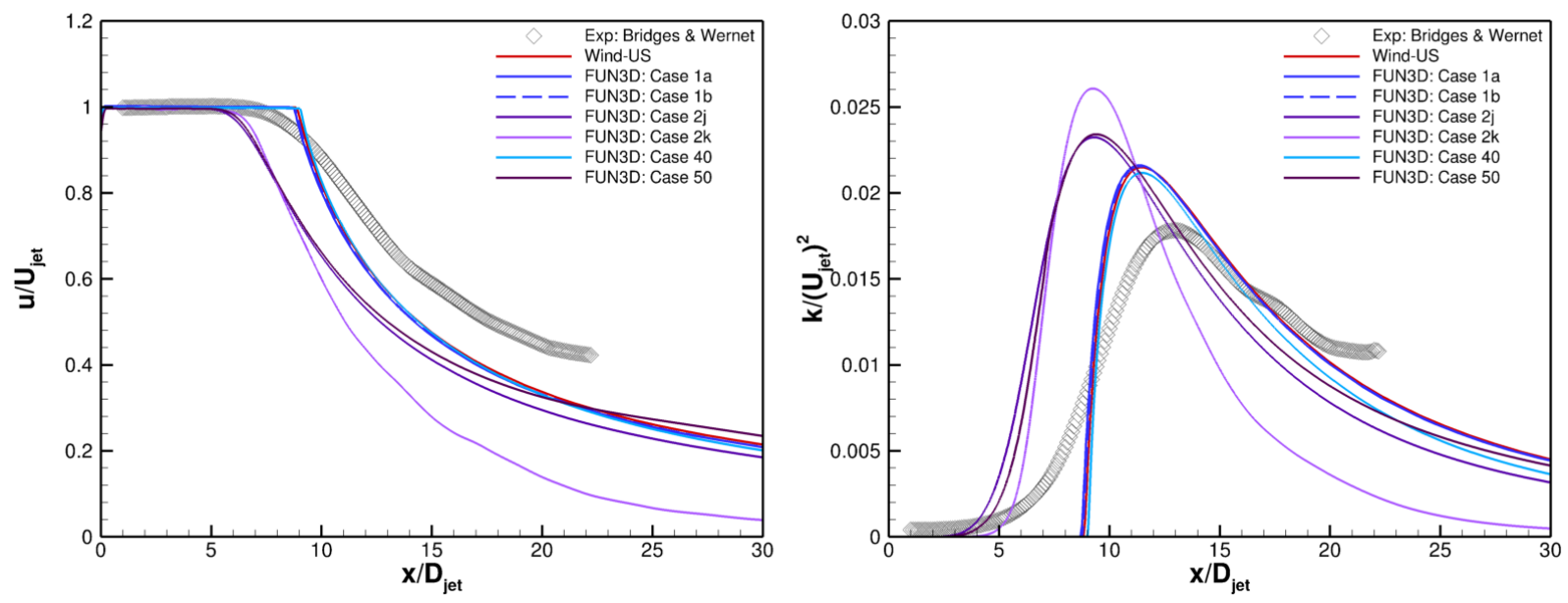

Figure 25.-Comparison of streamwise velocity, $u / U_{\text {jet }}$, and turbulent kinetic energy, $k /\left(U_{j e t}\right)^{2}$, along the centerline for experimental data and RANS solutions using the SST-V turbulence model.

\subsection{Triangular Prism Grids}

The Case $6 \mathrm{a}$ and Case $6 \mathrm{~b}$ grids were composed of triangular prism elements, generated by diagonalizing the baseline 2D axisymmetric structured grid. These grids provided an intermediate step between grids composed of structured-like hexahedral elements and grids composed of unstructured tetrahedral elements. The streamwise velocity and turbulent kinetic energy along the jet plume centerline are plotted in Figure 26 for the Case 6a and Case 6b grid simulations. The jet potential core of the Case 6a and Case $6 \mathrm{~b}$ grid simulations begins decaying $0.54 \times D_{j e t}$ and $0.37 \times D_{j e t}$ earlier, respectively, than the 
baseline Wind-US simulation. The peak turbulent kinetic energy along the centerline for the Case 6a and Case $6 \mathrm{~b}$ simulations occur $0.44 \times D_{\text {jet }}$ and $0.27 \times D_{\text {jet }}$ upstream, respectively, of that predicted by the baseline Wind-US simulation. Overall, there is good agreement between the simulations using the diagonalized structured grids and the baseline structured grid simulation-much better agreement than between the fully tetrahedral unstructured grids and the baseline structured grid simulation. This result indicates that a structured-like grid—a grid with structured-like flux faces—is necessary to obtain good results from RANS simulation of jet flows.

\subsection{Spalart-Allmaras Turbulence Model}

The previously discussed simulations all used Menter's two-equation Shear Stress Transport (SST-V) turbulence model. The following grids were also run using the S-A model: Case 1b, Case 2j, Case 50, Case 6a, and Case 6b. The streamwise velocity along the centerline is plotted in Figure 27 for these simulations. As with the simulations performed with the SST-V turbulence model, the simulations using structured-like grids (Case 1b, Case 6a, and Case 6b) show excellent agreement with the baseline Wind-US S-A solution (Ref. 6). Neither simulation performed using the fully tetrahedral element grids (Case 2j and Case 50) showed good agreement with the baseline Wind-US S-A solution. Interestingly though, whereas the fully tetrahedral element grid simulations predicted jet core decay earlier than the baseline when using the SST-V turbulence model, the same grids predicted a jet core decay later than the baseline when using the S-A turbulence model; $2.68 \times D_{\text {jet }}$ longer for Case $2 \mathrm{j}$ and $0.78 \times D_{\text {jet }}$ longer for Case 50. It is also interesting to note that there is a greater difference in jet core length $\left(1.90 \times D_{j e t}\right.$ Vs. $0.21 \times D_{j e t}$ ) for Case $2 \mathrm{j}$ and Case 50 when using the S-A turbulence model than when using the SST-V turbulence model. Whereas the S-A turbulence model affects simulations on fully unstructured grids differently than the SST-V turbulence model (e.g., jet core decayed later than baseline rather than earlier), the S-A simulations, like the SST-V simulations, show that structured-like grids produce better jet flow results than fully unstructured grids.
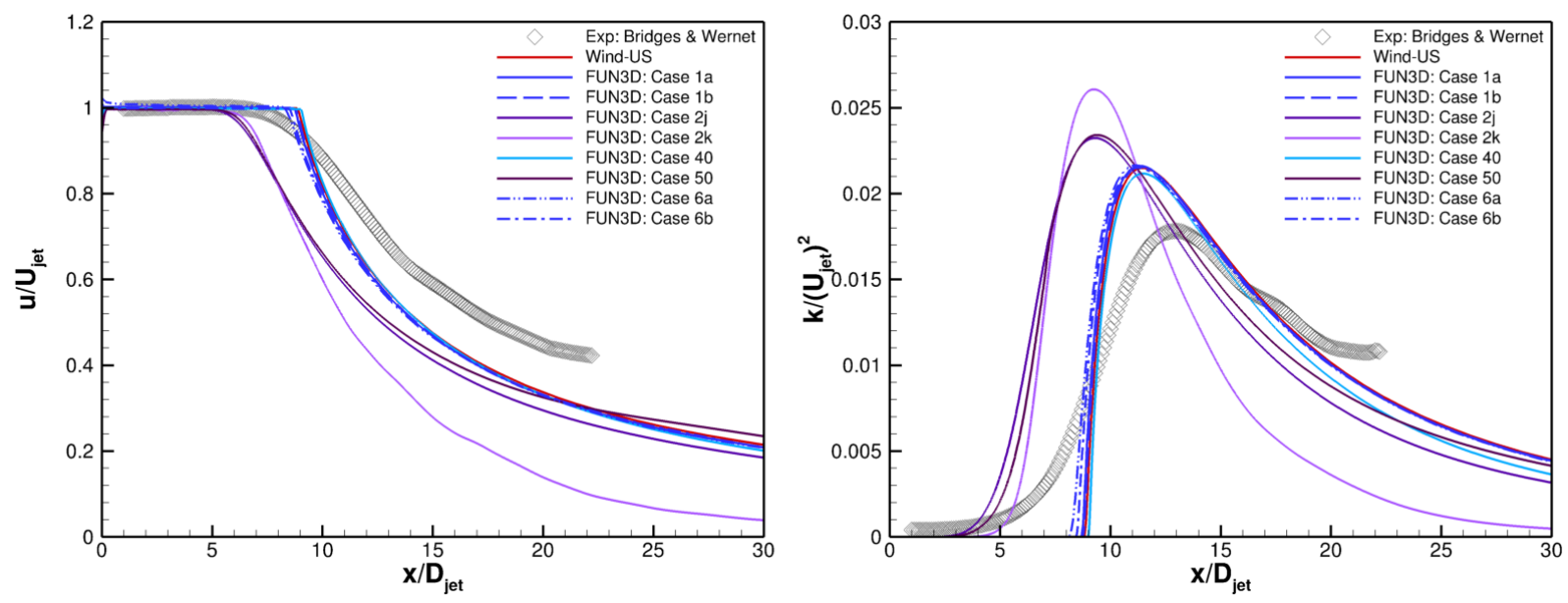

Figure 26.-Comparison of streamwise velocity, $u / U_{j e t}$, and turbulent kinetic energy, $k /\left(U_{\text {jet }}\right)^{2}$, along the centerline for experimental data and RANS solutions using the SST-V turbulence model. 


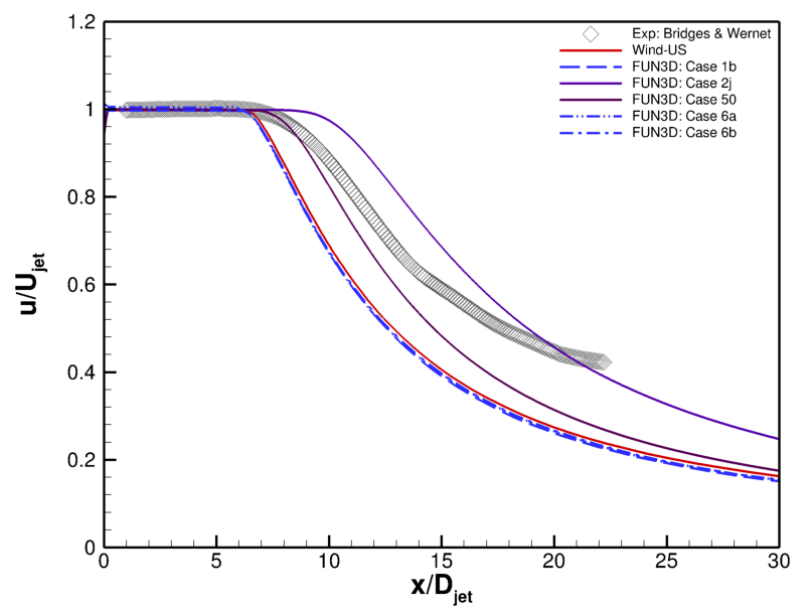

Figure 27.-Comparison of streamwise velocity, $u / \bigcup_{j e t}$, along the centerline for experimental data and RANS solutions using the Spalart-Allmaras turbulence model.
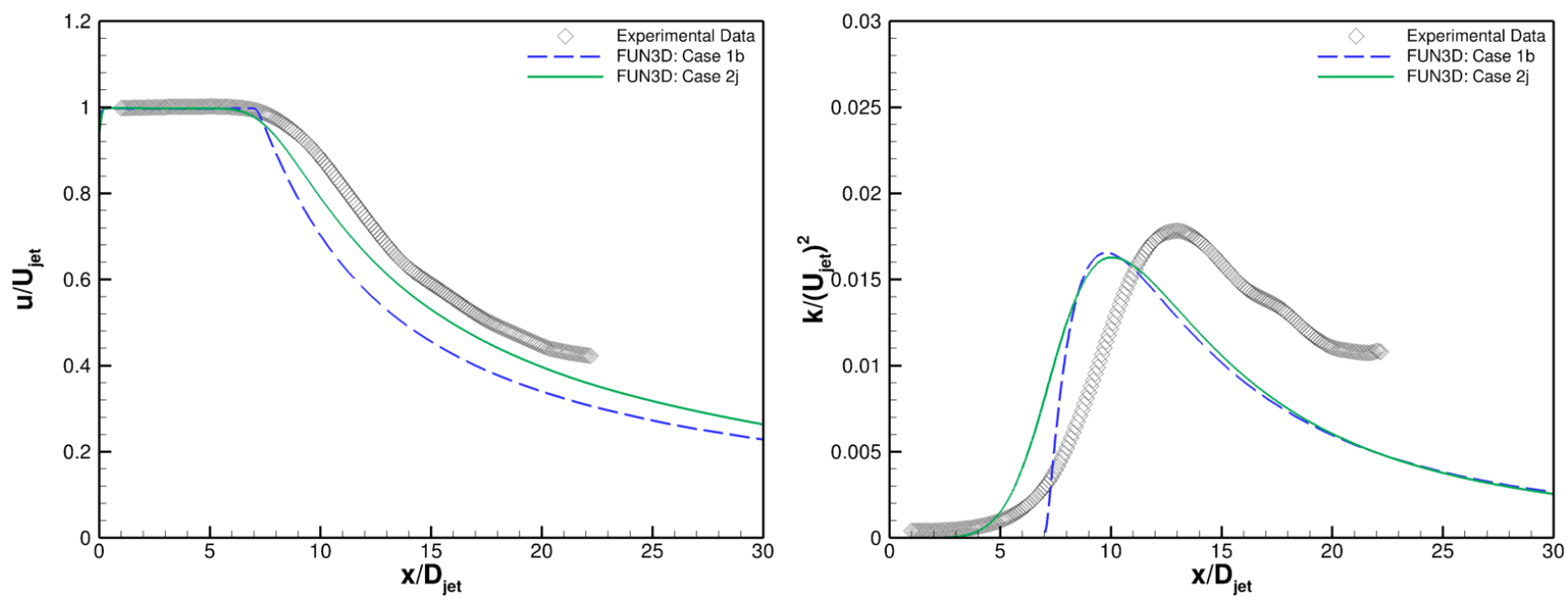

Figure 28.-Comparison of streamwise velocity, $u / U_{j e t}$, and turbulent kinetic energy, $k /\left(U_{j e t}\right)^{2}$, along the centerline for experimental data and RANS solutions using the $k-k L$ turbulence model.

\section{$5.9 \quad k-k L$ Turbulence Model}

The $k$ - $k L$ turbulence model was also used for simulations of the Case $1 \mathrm{~b}$ and Case $2 \mathrm{j}$ grids. The streamwise velocity and turbulent kinetic energy along the centerline are presented in Figure 28. There is currently no baseline $k-k L$ simulation data to compare with on the Turbulence Modeling Resource. However, since the prior simulations using the Case $1 \mathrm{~b}$ grid with the SST-V and S-A turbulence models showed good agreement with the baseline solutions, it is assumed the same would hold true for the $k$ - $k L$ turbulence model. The structured grid (Case 1b) and unstructured grid (Case 2j) solutions using the $k-k L$ model are in closer agreement than the solutions using the SST-V and S-A turbulence models are to their respective baseline solutions. The plot of centerline velocity shows that the jet potential core of the unstructured grid solution (Case $2 \mathrm{j}$ ) begins decaying about $0.7 \times D_{\text {jet }}$ earlier than the structured grid solution (Case 1b). This is similar to the behavior observed with the SST-V turbulence model solutions. However, the jet core of the unstructured grid solution decays more gradually than the structured grid solution. The plot of turbulent kinetic energy along the jet centerline shows that whereas the jet core of 
the unstructured grid solution does begin earlier, the peak value of turbulent kinetic energy and its location agree well with the structure grid solution. Whereas the jet simulations using the SST-V and S-A turbulence models appear to be quite sensitive to the grid topology, the solutions using the $k-k L$ turbulence model appear to be somewhat less sensitive.

\subsection{Comparison of Nozzle Performance}

The goal of this study is to determine best practices for generating unstructured grids for jet flows. So far, the jet plume flowfield (e.g., velocity and turbulent kinetic energy) has been compared for the ARN2 at near-sonic conditions to judge the performance of various grids. It is also worthwhile to compare how well the simulations compute massflow and thrust, since these quantities are typically what industry is most concerned about. The discharge coefficient, $C_{d}$, and thrust coefficient, $C_{V}$, were computed as follows:

$$
\begin{gathered}
C_{d}=\frac{\int_{\theta}^{2 \pi}\left(\int_{0}^{R} \text { jet } \rho \cdot u \cdot r \cdot d r\right) \cdot d \theta}{\rho_{\text {jet }} \cdot U_{j e t} \cdot A_{j e t}} \\
C_{V}=\frac{\int_{0}^{2 \pi}\left(\int_{0}^{R_{j e t}}\left[\rho \cdot u^{2} \cdot\left(p-p_{\infty}\right)\right] \cdot r \cdot d r\right) \cdot d \theta}{U_{j e t} \cdot \int_{0}^{2 \pi}\left(\int_{0}^{R} \text { jet } \rho \cdot u \cdot r \cdot d r\right) \cdot d \theta}
\end{gathered}
$$

The discharge and thrust coefficients are shown in Table II for the simulations using the SST-V turbulence model. The discharge coefficient for the baseline structured grid case was 0.992 . The simulations using a structured-like grid in the jet plume vary from 0.986 to 0.991 , whereas the simulations using an unstructured grid vary from 0.973 to 0.991 . The thrust coefficient of the baseline structured grid case was 0.995 . There was much less difference in the thrust coefficient among the unstructured grid simulations: less than \pm 0.002 , with many simulations within \pm 0.001 . The thrust coefficient uses the actual massflow rate in the denominator. As a result, on an absolute basis, the thrust force (in pounds-force or newtons, for example) varies as much as the discharge coefficient.

In addition to comparing the discharge and thrust coefficients, it is also important to consider how well the numerical simulations conserve massflow through the nozzle. Table II also shows the massflow conservation error between the nozzle inflow boundary and the nozzle exit for the SST-V simulations. The massflow conservation error is defined as follows:

$$
\text { Massflow Conservation Error }=\left|\frac{\text { Actual Inflow Massflow-Actual Exit Massflow }}{\text { Ideal Massflow }}\right|
$$

This shows that the structured-like grids conserved massflow through the nozzle the best, with an error in massflow no more than 0.08 percent. However, most of the unstructured grids did not conserve massflow as well, having massflow conservation errors ranging as high as 0.37 percent. The unstructured grid simulations that did have lower errors in massflow conservation (e.g., Case 20, Case 2a, and Case 2k), used grids that produced plume solutions that were not close in appearance to the baseline solution. These simulations show that a good grid for massflow and a good grid for jet plume flowfield do not necessarily coincide: different grid topology strategies are required to obtain the performance of structure-like grids. 
TABLE II.-COMPARISON OF MASSFLOW AND THRUST PERFORMANCE OF SIMULATIONS USING THE SST-V TURBULENCE MODEL

\begin{tabular}{|c|c|c|c|c|}
\hline Grid & $C_{d}$ & $C_{V}$ & $\begin{array}{c}\text { Massflow } \\
\text { conservation error }\end{array}$ & $\begin{array}{c}\text { Actual inflow } \\
\text { massflow/ideal massflow }\end{array}$ \\
\hline Wind-US & 0.992 & 0.995 & 0.0000 & 0.992 \\
\hline Case 1a & 0.990 & 0.996 & 0.0002 & 0.990 \\
\hline Case 1b & 0.990 & 0.995 & 0.0002 & 0.990 \\
\hline Case 1c & 0.986 & 0.995 & 0.0006 & 0.986 \\
\hline Case 20 & 0.989 & 0.995 & 0.0007 & 0.990 \\
\hline Case 2a & 0.989 & 0.995 & 0.0007 & 0.990 \\
\hline Case 2b & 0.975 & 0.995 & 0.0025 & 0.977 \\
\hline Case 2c & 0.975 & 0.995 & 0.0023 & 0.977 \\
\hline Case 2d & 0.975 & 0.995 & 0.0023 & 0.977 \\
\hline Case 2e & 0.991 & 0.995 & 0.0002 & 0.990 \\
\hline Case $2 \mathrm{f}$ & 0.990 & 0.995 & 0.0001 & 0.990 \\
\hline Case 2g & 0.976 & 0.995 & 0.0027 & 0.979 \\
\hline Case 2h & 0.976 & 0.995 & 0.0019 & 0.978 \\
\hline Case $2 \mathrm{i}$ & 0.976 & 0.995 & 0.0019 & 0.978 \\
\hline Case $2 \mathrm{j}$ & 0.975 & 0.995 & 0.0022 & 0.977 \\
\hline Case 2k & 0.973 & 0.995 & 0.0022 & 0.976 \\
\hline Case 30 & 0.973 & 0.995 & 0.0023 & 0.976 \\
\hline Case 3a & 0.973 & 0.995 & 0.0022 & 0.976 \\
\hline Case 3b & 0.973 & 0.995 & 0.0023 & 0.976 \\
\hline Case 3c & 0.978 & 0.993 & 0.0031 & 0.981 \\
\hline Case 3d & 0.990 & 0.996 & 0.0005 & 0.990 \\
\hline Case 40 & 0.990 & 0.996 & 0.0002 & 0.990 \\
\hline Case 50 & 0.978 & 0.994 & 0.0037 & 0.982 \\
\hline Case6a & 0.991 & 0.996 & 0.0008 & 0.992 \\
\hline Case6b & 0.991 & 0.995 & 0.0006 & 0.992 \\
\hline
\end{tabular}

The final column of Table II shows the ratio of the actual inflow massflow to the ideal massflow. The baseline Wind-US solution had an actual inflow massflow 0.8 percent less than the ideal massflow. The FUN3D solutions had inflow massflows ranging from 0.8 to 2.4 percent less than the ideal massflow, but the levels correspond well with the discharge coefficient, $C_{d}$. In an attempt to determine the cause of the differences in inflow massflow between the simulations, the streamwise velocity is plotted at the nozzle inflow in Figure 29 for several selected cases. The FUN3D solutions were selected to represent low (Case 2d and Case 2i), mid (Case 2j), and high (Case 1b) actual inflow massflows. Figure 29(a) shows that the baseline Wind-US solution has a more developed boundary layer at the nozzle inflow, while the FUN3D solutions have a less-developed boundary layer at the inflow. This suggests that the baseline Wind-US solution has less massflow at the nozzle inflow. However, as observed in Figure 29(b), the baseline Wind-US solution and the Case 1b solution have a greater velocity than the other simulations in the core region of the nozzle at the inflow. The plots of streamwise velocity in Figure 29 suggest that the reason for the differences in actual inflow massflow between the various solutions is more complicated than the low massflow solutions having a thicker inflow boundary layer.

The discharge coefficient and thrust coefficient for simulations using the S-A and $k$ - $k L$ turbulence models are shown in Table III and Table IV, respectively. As with the SST-V simulations, the structured- 
like grid simulations using the S-A turbulence model more closely agreed with the baseline structured grid solution than the unstructured grid solutions. While there was no baseline structured grid solution to compare the $k-k L$ simulations to, the solutions readily show that the structured grid simulation had a greater discharge coefficient and a greater thrust coefficient than the unstructured grid simulation. These differences are comparable to the differences observed between the structured and unstructured grid simulations performed with the SST-V and S-A turbulence models. The massflow conservation error and the ratio of actual massflow to ideal massflow are also listed in Table III and Table IV for the S-A and $k$ $k L$ turbulence model simulations, respectively. The trends follow what was observed for the SST-V turbulence model simulations. In general, the structured-like grid simulations produced discharge and thrust coefficients that were closer to the baseline structured grid simulations. The structured-like grid simulations also consistently did a better job conserving massflow through the nozzle.

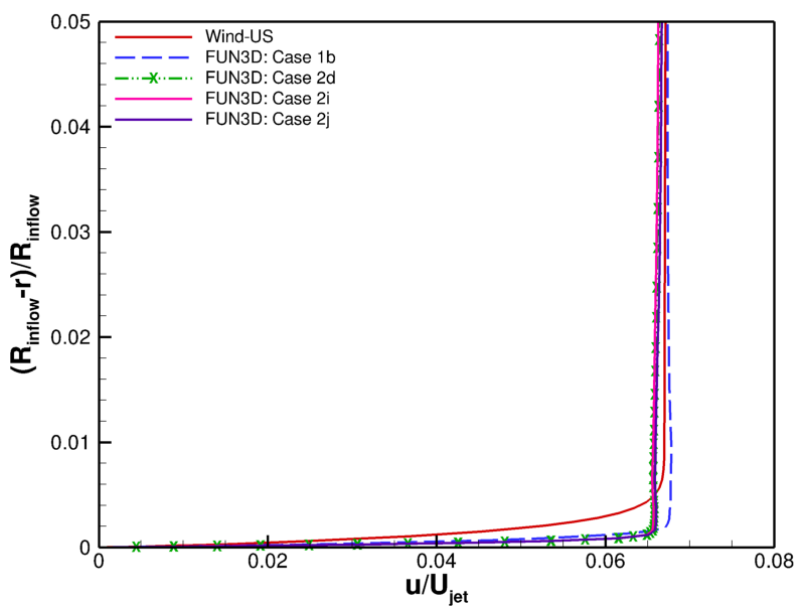

(a) Boundary layer.

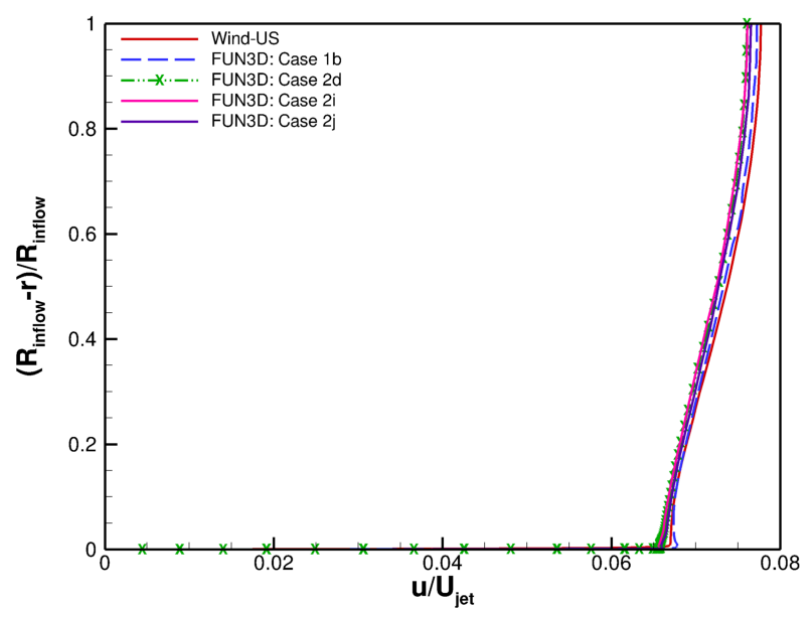

(b) Full nozzle inflow radius.

Figure 29.-Streamwise velocity at nozzle inflow using the SST-V turbulence model.

TABLE III.-COMPARISON OF MASSFLOW AND THRUST PERFORMANCE OF SIMULATIONS USING THE SPALART-ALLMARAS TURBULENCE MODEL

\begin{tabular}{|l|c|c|c|c|}
\hline \multicolumn{1}{|c|}{ Grid } & $C_{d}$ & $C_{V}$ & $\begin{array}{c}\text { Massflow } \\
\text { conservation error }\end{array}$ & $\begin{array}{c}\text { Actual inflow } \\
\text { massflow/ideal massflow }\end{array}$ \\
\hline Wind-US & 0.991 & 0.995 & 0.0001 & 0.991 \\
\hline Case 1b & 0.989 & 0.995 & 0.0004 & 0.990 \\
Case 2j & 0.977 & 0.993 & 0.0031 & 0.980 \\
Case 50 & 0.977 & 0.993 & 0.0027 & 0.980 \\
Case 6a & 0.991 & 0.995 & 0.0010 & 0.992 \\
Case 6b & 0.991 & 0.995 & 0.0007 & 0.991 \\
\hline
\end{tabular}

TABLE IV.-COMPARISON OF MASSFLOW AND THRUST PERFORMANCE OF SIMULATIONS USING THE K-KL TURBULENCE MODEL

\begin{tabular}{|c|c|c|c|c|}
\hline Grid & $C_{d}$ & $C_{V}$ & $\begin{array}{c}\text { Massflow } \\
\text { conservation error }\end{array}$ & $\begin{array}{c}\text { Actual inflow } \\
\text { massflow/ideal massflow }\end{array}$ \\
\hline Case 1b & 0.989 & 0.997 & 0.0005 & 0.989 \\
\hline Case 6b & 0.978 & 0.994 & 0.0034 & 0.981 \\
\hline
\end{tabular}




\subsection{Conclusions and Recommendations}

Over 30 simulations were completed using FUN3D, with 24 different grids, in order to determine the best practices for generating unstructured grids for Reynolds-Averaged Navier-Stokes (RANS) simulations of jet flows. The 2-in. diameter Acoustic Reference Nozzle (ARN2) - a round, single stream, convergent nozzle - with a near-sonic flow $\left(M_{j e t}=0.985, M_{a}=0.9\right)$ was used for this study. The results were judged against solutions of structured grid simulations provided by the Turbulence Modeling Resource. Simulations were performed with all the grids using Menter's Shear Stress Transport (SST-V) turbulence model; simulations were performed on several select grids using the Spalart-Allmaras (S-A) turbulence model and the $k-k L$ turbulence model.

For the near-sonic ARN2 jet flow, RANS simulations using the SST-V turbulence model performed on unstructured grids composed primarily of tetrahedral and pentahedral elements consistently predicted an earlier jet core decay than the baseline structured grid solution. The closest a fully unstructured grid solution came to matching the baseline structured grid solution was by predicting the jet core to begin decaying $3.4 \times D_{\text {jet }}$ upstream of the baseline solution. Consequently, the peak turbulent kinetic energy along the centerline was also predicted no less than $2.0 \times D_{\text {jet }}$ upstream of that of the baseline solution, at values at least 8.1 percent greater. For simulations using the S-A turbulence model, the fully unstructured grids predicted the jet core decay later than the baseline structured grid simulation. Using the $k-k L$ turbulence model, the fully unstructured grid produced a jet core that had an earlier initial breakdown but a more gradual decay than the structured grid. The $k$ - $k L$ turbulence model appears to be somewhat less sensitive to the grid topology (e.g., structured-like vs. unstructured) than the SST-V and S-A turbulence models, as observed in the good agreement in centerline turbulent kinetic energy (beginning at the peak). Nozzle massflow and thrust performance were also investigated. For all turbulence models, the unstructured grid simulations generally had massflows that were up to 1.9 percent less than the baseline structured grid simulations, which was more than the simulations performed with structured-like grids. Thrust performance was generally better, deviating from the baseline solution by less than \pm 0.2 percent. Massflow conservation between the nozzle inflow and exit were compared. Most unstructured grid solutions-including all those with the better jet plume predictions-lost between 0.19 and 0.37 percent of the massflow in the nozzle.

Simulations using a structured-like grid through the entire jet plume consistently showed good agreement with the baseline structured grid solution. This applies to aligned hexahedral elements and aligned triangular prism elements. The result was true for the SST-V and S-A turbulence models (there was no baseline to compare against for the $k-k L$ turbulence model). The simulations with structured-like grids produced nozzle massflows closer to the baseline solutions than the unstructured grids, no more than 0.06 percent less than the baseline solution. Thrust performance of the structured-like grids was similar in nature to the unstructured grids, varying less than \pm 0.2 percent from the baseline solutions. Additionally, the structured-grid simulations conserved massflow through the nozzle within 0.08 percent. Since this study found that RANS simulations using structured-like grids were in better agreement with the baseline structured grid simulations, it is recommended that aligned, structured-like grid elements be used in jet flows whenever possible.

\section{References}

1. Pao, S. P., Abdol-Hamid, K. S., Campbell, R. L., Hunter, C. A., Massey, S. J., and Elmiligui, A. A., Unstructured CFD and Noise Prediction Methods for Propulsion Airframe Aeroacoustics, AIAA Paper 2006-2597, May 2006. 
2. Kleb, B., Schauerhamer, D. G., Trumble, K., Sozer, E., Barnhardt, M., Carlson, J., and Edquist, K., Toward Supersonic Retropropulsion CFD Validation, AIAA Paper 2011-3490, June 2011.

3. Schauerhamer, D. G., Trumble, K. A., Kleb, B., Carlson, J., and Edquist, K., Continuing Validation of Computational Fluid Dynamics for Supersonic Retropropulsion, AIAA Paper 2012-0864, January 2012.

4. Zarhi, K. A., Schauerhamer, D. G., Kleb, W. L., Carlson, J., and Edquist, K. T., Analysis of NavierStokes Codes Applied to Supersonic Retropropulsion Wind-Tunnel Test, J. of Spacecraft and Rockets, Vol. 51, No. 3, May-June 2014, pp. 680-692.

5. Carter, M. B., Elmiligui, A. A., Campbell, R. L., and Nayani, S. N., USM3D Predictions of Supersonic Nozzle Flow, AIAA Paper 2014-2270, June 2014.

6. NASA Langley Research Center Turbulence Modeling Resource: Axisymmetric Near-Sonic Jet [webpage], URL: https://turbmodels.larc.nasa.gov/jetnearsonic_val.html, [cited 1 April 2018].

7. Bridges, J. and Wernet, M. P., Establishing Consensus Turbulence Statistics for Hot Subsonic Jets, AIAA Paper 2010-3751, June 2010.

8. Bridges, J. and Wernet, M. P., The NASA Subsonic Jet Particle Image Velocimetry (PIV) Dataset, NASA/TM-2011-216807, November 2011.

9. Yoder, D. A., Wind-US User’s Guide Version 3.0, NASA/TM-2016-219078, March 2016.

10. Menter, F. R., Two-Equation Eddy Viscosity Turbulence Models for Engineering Applications, AIAA Journal, Vol. 32, No. 8 (1994), pp. 1598-1605. doi: 10.2514/3.12149.

11. Spalart, P. R. and Allmaras, S. R., A One-Equation Turbulence Model for Aerodynamic Flows, Recherche Aerospatiale, No. 1, 1994, pp. 5-21.

12. Jespersen, D. C., Pulliam, T. H., and Childs, M. L., OVERFLOW Turbulence Modeling Resource Validation Results, NAS Technical Report NAS-2016-01, 2016.

13. Biedron, R. T., Carlson, J., Derlaga, J. M., Gnoffo, P. A., Hammond, D. P., Jones, W. T., Kleb, B., Lee-Rausch, E. M., Nielsen, E. J., Park, M. A., Rumsey, C. L., Thomas, J. L., and Wood, W. A., FUN3D Manual: 12.9, NASA/TM—2016-219012, Feb. 2016.

14. Roe, P. L., Approximate Riemann Solvers, Parameter Vectors, and Difference Schemes, Journal of Computational Physics, Vol. 43, 1981, pp. 357-372.

15. Van Leer, B., Flux-Vector Splitting for the Euler Equations, ICASE Report 82-30, 1982.

16. Abdol-Hamid, K. S., Carlson, J., and Rumsey, C. L., Verification and Validation of the k-kL Turbulence Model in FUN3D and CFL3D Codes, NASA/TM-2015-218968, Nov. 2015.

17. NASA High-End Computing Capability [webpage], URL: http://www.nas.nasa.gov/hecc/, [cited 1 April 2018].

18. Pointwise ${ }^{\circledR}$ [software package], Ver. 17.3 Release 4, Pointwise, Inc., Fort Worth, TX, 2015. 

